\title{
ON DIFFERENTIAL EQUATIONS ASSOCIATED WITH EULER PRODUCT EXPRESSIONS
}

BY

IAN KNOWLES

\begin{abstract}
A method is given by which one may associate (uniquely) certain differential equations with analytic functions defined by certain Euler product expressions. Some of the consequences of this construction include results relating the location of the zeros of the analytic functions to asymptotic properties of the solutions of the differential equations, as well as a differential equation characterization of those Dirichlet series with multiplicative coefficients.
\end{abstract}

1. Introduction. Let $\left\{d_{n}\right\}_{n \geqslant 1}$ and $\left\{\lambda_{n}\right\}_{n \geqslant 1}$ be given sequences of real numbers satisfying

$$
d_{n}>-1, \quad d_{n} \neq 0, \quad \lambda_{n}>1 \quad \text { for } n \geqslant 1,
$$

and

$$
\sum_{n=1}^{\infty}\left|d_{n}\right|<\infty,
$$

and consider the formal Euler product expression

$$
E(s)=\prod_{n=1}^{\infty}\left(1+\frac{d_{n}}{\lambda_{n}^{s}}\right) .
$$

If the sequences $\left\{d_{n}\right\}$ and $\left\{\lambda_{n}\right\}$ are suitably chosen, then the Euler product (1.3) will converge absolutely in some right half-plane $\operatorname{Re}(s)>\sigma_{0}$ and define there an analytic function. In many cases (though not always-see, e.g., [10]) this function $E(s)$ has an analytic continuation, also denoted by $E(s)$, to a larger domain. For example, if $\left\{p_{n}\right\}$ denotes the set of prime numbers listed in increasing order beginning with $p_{1}=2$, and we set

$$
d_{n}=p_{n}^{-\theta}, \quad \lambda_{n}=p_{n},
$$

where $\theta>1$ is fixed, then $\left[13\right.$, p. 5] $E(s)=\zeta(s+\theta)(\zeta(2 s+2 \theta))^{-1}$, where $\zeta(s)$ denotes the Riemann zeta function; if

$$
d_{n}=-p_{n}^{-\theta}, \quad \lambda_{n}=p_{n},
$$

Received by the editors October 31, 1983 and, in revised form, June 1, 1984.

1980 Mathematics Subject Classification. Primary 30C15; Secondary 34B05, 35L20, 10 H05. 
then $E(s)=(\zeta(s+\theta))^{-1}$; finally, if

$$
d_{n}=-(\pi n)^{-2}, \quad \lambda_{n}=e^{2}, \quad z=e^{-s},
$$

then [14, p. 114] $E(s)=z^{-1} \sin z$.

For the case (1.4) above, it was shown in [4] that one can associate with the Euler product (1.3) a second-order differential equation $[4,(4.6)]$ arising from a sequence of eigenvalue problems [4, (3.4)-(3.5)] associated with the partial products of (1.3). Our main intention here is to extend this process to cover the more general Euler product (1.3) and to investigate in detail some of the consequences of this construction. Included here are a proof $(\S \S 2,3)$ that the differential equations are uniquely determined by the Euler products and the fact that such differential equations and their associated eigenfunctions may be used to characterize those Dirichlet series $\sum_{n=1}^{\infty} a_{n} n^{-s}$ that have Euler products $\prod_{n=1}^{\infty}\left(1+d_{n} p_{n}^{-s}\right)$. This is somewhat comparable to the Hecke-Petersson modular form characteriztion, although the functional equation aspect of the latter is not apparent as yet. It is possible that by considering higher-order differential operators, one can similarly represent more complicated Euler products, but this remains to be seen. In $\$ 4$ we consider some properties relating the (limiting) differential equation, its corresponding integral equation, and the zeros of the analytic function $E(s)$, and in $\$ 5$ we briefly consider the formal connection between $E(s)$ and a certain hyperbolic partial differential equation resembling, in the special cases (1.4)-(1.5), the familiar automorphic wave equation [5].

2. Construction of the associated differential equations. Here we outline a modified version of the construction in [4]. The idea is as follows. Beginning with the Euler product (1.3), we write this as a series

$$
\prod_{n=1}^{\infty}\left(1+\frac{d_{n}}{\lambda_{n}^{s}}\right)=\prod_{n=1}^{\infty} \frac{\varepsilon^{*}(n)}{q(n)^{s}},
$$

where the numbers $q(n)$ and $\varepsilon^{*}(n)$ are formed from the sequences $\left\{\lambda_{n}\right\}$ and $\left\{d_{n}\right\}$, respectively, according to the formulae

$$
q(1)=1, \quad q(2)=\lambda_{1}, \quad q\left(2^{n}+k\right)=\lambda_{n+1} q(k), \quad 1 \leqslant k \leqslant 2^{n}, n \geqslant 1,
$$

$$
\varepsilon^{*}(1)=1, \quad \varepsilon^{*}(2)=d_{1}, \quad \varepsilon^{*}\left(2^{n}+k\right)=d_{n+1} \varepsilon^{*}(k), \quad 1 \leqslant k \leqslant 2^{n}, n \geqslant 1 .
$$

Notice that for $N \geqslant 1$,

$$
\prod_{n=1}^{N}\left(1+\frac{d_{n}}{\lambda_{n}^{s}}\right)=\sum_{n=1}^{2^{N}} \frac{\varepsilon^{*}(n)}{q(n)^{s}} .
$$

We next attempt to form an eigenvalue problem $E_{n}(n \geqslant 1)$ of the form

$$
\begin{gathered}
y^{\prime \prime}-s b_{n}(x) y^{\prime}+s^{2} c_{n}(x) y=0, \quad 0 \leqslant x<\infty, \\
y(0)=0, \\
y(x) \sim e^{\beta_{n+1} s x} \text { as } x \rightarrow \infty
\end{gathered}
$$


in such a way that the equation for the eigenvalues is of the form

$$
\sum_{k=1}^{2^{n}} \frac{\varepsilon^{*}(k)}{q(k)^{s}}=0 .
$$

Here, for each $n, \beta_{n+1}$ is a suitably chosen constant, and the functions $b_{n}(x)$ and $c_{n}(x)$ are assumed to be step fucntions defined on a certain infinite partition $0=a_{0}<a_{1}<\cdots$ of $[0, \infty)$ by certain number sequences $\left\{b_{r}\right\}_{r \geqslant 1}$ and $\left\{c_{r}\right\}_{r \geqslant 1}$ according to the formulae

$$
\begin{aligned}
b_{n}(x) & =b_{r}, \quad a_{r-1} \leqslant x<a_{r}, \quad 1 \leqslant r \leqslant n, \\
& =b_{n+1}, \quad x \geqslant a_{n}, \\
c_{n}(x) & =c_{r}, \quad a_{r-1} \leqslant x<a_{r}, \quad 1 \leqslant r \leqslant n, \\
& =c_{n+1}, \quad x \geqslant a_{n} .
\end{aligned}
$$

Observe that the same partition $\left\{a_{r}\right\}_{r \geqslant 0}$ and number sequences $\left\{b_{r}\right\}$ and $\left\{c_{r}\right\}$ serve to define each of the functions $b_{n}(x)$ and $c_{n}(x), n \geqslant 1$. Due to degeneracies in the resulting equations for the numbers $\beta_{r}, a_{r}, b_{r}$ and $c_{r}$, one cannot choose these numbers to obtain precisely (2.8) as the eigenvalue equation. However, by choosing a sequence $\delta=\left\{\delta_{1}, \delta_{2}, \ldots\right\}$ satisfying

$$
\delta_{r} d_{r}>0, \quad r \geqslant 1,
$$

but otherwise arbitrary for the moment, one can choose $\left\{a_{r}\right\},\left\{b_{r}\right\},\left\{c_{r}\right\}$, and $\left\{\beta_{r}\right\}$ (now depending on $\delta$ ) so that the eigenvalue equation for (2.5)-(2.7) has the form

$$
\left[\sum_{k=1}^{2^{n}} \frac{\varepsilon^{*}(k)}{q(k)^{s}}\right] \chi_{n}(0, s ; \delta)=0
$$

where $\chi_{n}(x, s ; \delta)$ is a certain solution of $(2.5)$ for which $\chi_{n}(0, s ; 0) \neq 0$ for all $n, s$ (see (2.78)); thus (2.8) is essentially the case $\delta=0$ in (2.11).

The precise formulae for the constants in (2.5)-(2.7) can be obtained by a somewhat tedious process of trial and error involving the solutions of (2.5). For simplicity we proceed by stating the appropriate formulae and then deriving their properties directly.

Define the sequence $\varepsilon(n)$ by

$$
\varepsilon(1)=1, \quad \varepsilon(2)=d_{1},
$$

and, for $n \geqslant 1$,

$$
\begin{aligned}
\varepsilon\left(2^{n}+k\right) & =d_{n+1} \varepsilon(k), \quad 1 \leqslant k \leqslant 2^{n-1}, \\
& =\left(1+\delta_{n}\right) d_{n+1} \varepsilon(k), \quad 1+2^{n-1} \leqslant k \leqslant 2^{n} .
\end{aligned}
$$

Observe that on setting $d_{n}=p_{n}^{-\theta}$ in [4, Lemma 2.1] we have

LEMMA 2.1. For integers $n \geqslant 1$ and $q \geqslant 0$,

$$
\varepsilon\left(1+2^{n-1}+q \cdot 2^{n+1}\right)=d_{n} \varepsilon\left(1+q \cdot 2^{n+1}\right),
$$

(ii) $\varepsilon\left(1+3 \cdot 2^{n-1}+q \cdot 2^{n+1}\right)=\left(1+\delta_{n}\right) d_{n} \varepsilon\left(1+2 \cdot 2^{n-1}+q \cdot 2^{n+1}\right)$.

Next, define the sequence $\left\{w_{n}^{(r)}\right\}, n \geqslant 0,0 \leqslant r \leqslant n$, by

$$
w_{n}^{(r)}=\varepsilon(1)+\varepsilon\left(1+2^{r}\right)+\cdots+\varepsilon\left(1+k \cdot 2^{r}\right)+\cdots+\varepsilon\left(1+2^{n}-2^{r}\right)
$$


and set $w_{n}^{(0)}=w_{n}$. Observe that

$$
w_{n}^{(n-1)}=\varepsilon(1)+\varepsilon\left(1+2^{n-1}\right)=1+d_{n}, \quad w_{n}^{(n)}=\varepsilon(1)=1 .
$$

We also have

LEMMA 2.2. For $n \geqslant 3$ and $n \geqslant r+2$,

$$
\begin{gathered}
w_{n}^{(r)}=\left(1+d_{n}\right) w_{n-1}^{(r)}+\delta_{n-1} d_{n}\left(w_{n-1}^{(r)}-w_{n-2}^{(r)}\right), \\
d_{n}^{-1}\left(w_{n}^{(r)}-w_{n-1}^{(r)}\right)=w_{n-1}^{(r)}+\delta_{n-1}\left(w_{n-1}^{(r)}-w_{n-2}^{(r)}\right) .
\end{gathered}
$$

This is essentially [4, Lemma 2.1]. Define $K_{r}^{(n)}$ by $K_{r}^{(r+1)}=1$ and for $n \geqslant r+2$,

$$
K_{r}^{(n)}=\frac{d_{n}^{-1}\left[w_{n}^{(r)}-w_{n-1}^{(r)}\right]}{\left(1+d_{r+1}\right)\left(1+d_{r+2}\right) \cdots\left(1+d_{n-1}\right)} .
$$

Then we have

LEMMA 2.3. (i) For $n \geqslant r+2$,

(ii)

$$
\begin{gathered}
w_{n}^{(r)}=\prod_{i=r+1}^{n}\left(1+d_{i}\right) \cdot\left[1+\sum_{j=r+1}^{n-1} \frac{\delta_{j} K_{r}^{(j)}}{\left(1+d_{j}^{-1}\right)\left(1+d_{j+1}^{-1}\right)}\right] ; \\
K_{r}^{(r+2)}=1+\delta_{r+1} /\left(1+d_{r+1}^{-1}\right)
\end{gathered}
$$

and, for $n \geqslant r+3$,

$$
K_{r}^{(n)}=1+\sum_{j=r+1}^{n-2} \frac{\delta_{j} K_{r}^{(j)}}{\left(1+d_{j}^{-1}\right)\left(1+d_{j+1}^{-1}\right)}+\frac{\delta_{n-1} K_{r}^{(n-1)}}{\left(1+d_{n-1}^{-1}\right)} .
$$

Proof. (i) From Lemma 2.2(i) and (2.15),

$$
\begin{aligned}
w_{r+2}^{(r)} & =\left(1+d_{r+2}\right) w_{r+1}^{(r)}+\delta_{r+1} d_{r+2}\left(w_{r+1}^{(r)}-w_{r}^{(r)}\right) \\
& =\left(1+d_{r+2}\right)\left(1+d_{r+1}\right)+\delta_{r+1} d_{r+1} d_{r+2} \\
& =\left(1+d_{r+1}\right)\left(1+d_{r+2}\right)\left[1+\delta_{r+1} /\left(1+d_{r+1}^{-1}\right)\left(1+d_{r+2}^{-1}\right)\right] .
\end{aligned}
$$

For $n \geqslant r+3$ one can show by induction (and Lemma 2.2(i)) that

$$
\begin{aligned}
w_{n}^{(r)}= & \left(1+d_{r+1}\right)\left(1+d_{r+2}\right) \cdots\left(1+d_{n}\right) \\
& +\delta_{r+1} d_{r+1} d_{r+2}\left(1+d_{r+3}\right) \cdots\left(1+d_{n}\right) \\
& +\delta_{r+2} d_{r+2} d_{r+3}\left[d_{r+2}^{-1}\left(w_{r+2}^{(r)}-w_{r+1}^{(r)}\right)\right]\left(1+d_{r+4}\right) \cdots\left(1+d_{n}\right) \\
& \quad \vdots \\
& +\delta_{n-1} d_{n-1} d_{n}\left[d_{n}^{-1}\left(w_{n-1}^{(r)}-w_{n-2}^{(r)}\right)\right] .
\end{aligned}
$$

Consequently,

$$
w_{n}^{(r)}=\left(1+d_{r+1}\right) \cdots\left(1+d_{n}\right)\left[1+\sum_{j=r+1}^{n-1} \frac{\delta_{j} K_{r}^{(j)}}{\left(1+d_{j}^{-1}\right)\left(1+d_{j+1}^{-1}\right)}\right]
$$

as required. 
(ii) Set

$$
\Phi_{r}^{(n)}=w_{n}^{(r)}\left[\prod_{j=r+1}^{n}\left(1+d_{j}\right)\right]^{-1}
$$

Then from Lemma 2.2(ii),

$$
\begin{aligned}
K_{r}^{(n)} & =\Phi_{r}^{(n-1)}+\frac{\delta_{n-1} K_{r}^{(n-1)}}{1+d_{n-1}^{-1}} \\
& =\left[1+\sum_{j=r+1}^{n-2} \frac{\delta_{j} K_{r}^{(j)}}{\left(1+d_{j}^{-1}\right)\left(1+d_{j+1}^{-1}\right)}\right]+\frac{\delta_{n-1} K_{r}^{(n-1)}}{\left(1+d_{n-1}^{-1}\right)}
\end{aligned}
$$

by part (i) above.

LeMma 2.4. There exists a constant $\delta^{*}>0$ such that if $\sup \left\{\left|\delta_{r}\right|: r \geqslant 1\right\} \leqslant \delta^{*}$, then $K_{r}^{(n)}(\delta) \leqslant 2, w_{n}^{(r)}(\delta)>0$, and $\delta_{n}\left(w_{n}-w_{n-1}\right)>0$ for all $n, r$ with $0 \leqslant r \leqslant n$.

Proof. Observe first that, as a consequence of assuming (1.2), $d_{k} \rightarrow 0$ as $k \rightarrow \infty$, and the series $\Sigma\left[\left(1+d_{k}^{-1}\right)\left(1+d_{k+1}^{-1}\right)\right]^{-1}$ is absolutely convergent. Choose $\delta^{*}$ so that

$$
\delta^{*}\left[C+\sum_{k=1}^{\infty} \frac{1}{\left(1+d_{k}^{-1}\right)\left(1+d_{k+1}^{-1}\right)}\right] \leqslant \frac{1}{4},
$$

where $C=\sup \left\{\left|d_{k}\left(1+d_{k}\right)^{-1}\right|: k \geqslant 1\right\}$. To show that $K_{r}^{(n)}(\delta) \leqslant 2$ for

$$
\sup \left\{\left|\delta_{r}\right|: r \geqslant 1\right\} \leqslant \delta^{*} \text {, }
$$

we use induction on $n$ for fixed $r$.

Observe that by Lemma 2.3(ii),

$$
K_{r}^{(r+2)} \leqslant 1+\delta^{*} C \leqslant 2
$$

by (2.20). Also, assuming $K_{r}^{(m)} \leqslant 2$ for $r+2 \leqslant m \leqslant n-1$, we have from Lemma 2.3(ii) and (2.20) that

$$
K_{r}^{(n)} \leqslant 1+2 \delta^{*}\left(C+\sum_{k=1}^{\infty} \frac{1}{\left(1+d_{k}^{-1}\right)\left(1+d_{k+1}^{-1}\right)}\right) \leqslant 2,
$$

and thus $K_{r}^{(n)} \leqslant 2$ for all $n, r, 0 \leqslant r \leqslant n$. Next, observe that for all $n, r$, and $\left\{\delta_{r}\right\}$ with $\sup \left\{\left|\delta_{r}\right|: r \geqslant 1\right\} \leqslant \delta^{*}$, by $(2.20)$,

$$
\left|\sum_{j=r+1}^{n-1} \frac{\delta_{j} K_{r}^{(j)}}{\left(1+d_{j}^{-1}\right)\left(1+d_{j+1}^{-1}\right)}\right| \leqslant 2 \delta^{*} \sum_{k=1}^{\infty} \frac{1}{\left(1+d_{k}^{-1}\right)\left(1+d_{k+1}^{-1}\right)} \leqslant \frac{1}{2} .
$$

Consequently, by Lemma 2.3(i),

$$
w_{n}^{(r)} \geqslant \frac{1}{2} \prod_{j=r+1}^{n}\left(1+d_{j}\right)>0 .
$$

Finally, given that $w_{n}>0$ for all $n$, it is now not difficult to show by induction (and (2.10), Lemma 2.2(ii)) that $\delta_{n}\left(w_{n}-w_{n-1}\right)>0$ for all $n$.

We henceforth assume that the sequence $\left\{\delta_{r}\right\}$ is chosen so that

$$
\sup \left\{\left|\delta_{r}\right|: r \geqslant 1\right\} \leqslant \delta^{*},
$$

where $\delta^{*}$ satisfies (2.20). 
Next, define $\left\{\alpha_{r}\right\}_{r \geqslant 1},\left\{\beta_{r}\right\}_{r \geqslant 1}$ by

$$
\begin{aligned}
& \alpha_{1}=0, \quad \beta_{1}=-1, \\
& \alpha_{r}=-\left(w_{r}^{(1)}-w_{r-1}^{(1)}\right) /\left(w_{r}-w_{r-1}\right), \quad r \geqslant 2, \\
& \beta_{r}=-w_{r-1}^{(1)} / w_{r-1}, \quad r \geqslant 2 .
\end{aligned}
$$

These formula differ from [4, (2.10)-(2.13)] in that the factor $w_{n}$ has been omitted.

The following formulae will be of interest later.

LEMMA 2.5. For $r \geqslant 1$,

$$
\frac{\alpha_{r}-\beta_{r+1}}{\alpha_{r}-\beta_{r}}=\frac{w_{r-1}}{w_{r}}
$$

(ii)

$$
\frac{\beta_{r+1}-\beta_{r}}{\alpha_{r}-\beta_{r}}=\frac{w_{r}-w_{r-1}}{w_{r}}
$$

(iii)

$$
\frac{\alpha_{r}-\alpha_{r+1}}{\alpha_{r}-\beta_{r}}=\frac{d_{r+1} w_{r-1}}{w_{r+1}-w_{r}}
$$

(iv)

$$
\frac{\alpha_{r+1}-\beta_{r}}{\alpha_{r}-\beta_{r}}=\left(1+\delta_{r}\right) d_{r+1} \cdot \frac{w_{r}-w_{r-1}}{w_{r+1}-w_{r}} .
$$

This is essentially [4, Lemma 2.5].

LEMMA 2.6. (i) $\beta_{2}-\beta_{1}=d_{1} /\left(1+d_{1}\right)$;

(ii) for $r \geqslant 2$,

$$
\begin{gathered}
\alpha_{r}-\beta_{r}=\frac{1}{w_{r-1}\left[w_{r-1}+\delta_{r-1}\left(w_{r-1}-w_{r-2}\right)\right]} \prod_{k=1}^{r-1}\left(\delta_{k} d_{k}\right), \\
\beta_{r+1}-\beta_{r}=\frac{d_{r}}{w_{r-1} w_{r}} \prod_{k=1}^{r-1}\left(\delta_{k} d_{k}\right) .
\end{gathered}
$$

Proof. As (i) is obvious from the definition, and (2.26) follows from (2.25) and Lemma 2.5(ii), it suffices to prove (2.25). From Lemma 2.5 (i), (iii), and Lemma 2.2(ii),

$$
\begin{aligned}
\frac{\alpha_{r+1}-\beta_{r+1}}{\alpha_{r}-\beta_{r}} & =\frac{w_{r-1}}{w_{r}}-\frac{d_{r+1} w_{r-1}}{w_{r+1}-w_{r}} \\
& =\frac{w_{r-1}\left(w_{r+1}-w_{r}\right)-d_{r+1} w_{r} w_{r-1}}{w_{r}\left(w_{r+1}-w_{r}\right)} \\
& =\frac{w_{r-1}\left(w_{r}-w_{r-1}\right)}{w_{r}\left(w_{r+1}-w_{r}\right)} \cdot \delta_{r} d_{r+1} .
\end{aligned}
$$

Thus

$$
\begin{aligned}
\alpha_{r}-\beta_{r} & =\prod_{k=1}^{r}\left(\frac{\alpha_{k+1}-\beta_{k+1}}{\alpha_{k}-\beta_{k}}\right)=\prod_{k=1}^{r-1}\left(\delta_{k} d_{k}\right) \cdot \frac{d_{r}}{w_{r-1}\left(w_{r}-w_{r-1}\right)} \\
& =\prod_{k=1}^{r-1}\left(\delta_{k} d_{k}\right) \cdot \frac{1}{w_{r-1}\left[w_{r-1}+\delta_{r-1}\left(w_{r-1}-w_{r-2}\right)\right]}
\end{aligned}
$$


Finally, define $\left\{b_{r}\right\},\left\{c_{r}\right\}$ by

$$
b_{r}=\alpha_{r}+\beta_{r}, \quad c_{r}=\alpha_{r} \beta_{r}
$$

and $\left\{a_{n}\right\}$ by $a_{0}=0$ and

$$
a_{r}=a_{r-1}+\ln \left(\lambda_{r}\right) /\left(\alpha_{r}-\beta_{r}\right), \quad r \geqslant 1 .
$$

Observe that the partition $\left\{a_{r}\right\}$ is well defined as $\lambda_{r}>1$, and $\alpha_{r}-\beta_{r}>0$ by (2.10), (2.25), and the fact that $\delta_{n}\left(w_{n}-w_{n-1}\right)>0$ (Lemma 2.4). It is clear from (2.27) that for each $r$ the numbers $\alpha_{r}, \beta_{r}$ are just the characteristic roots for the constant coefficient equation (2.5) on $\left[a_{r-1}, a_{r}\right]$. Thus, for $x$ in $\left[a_{r-1}, a_{r}\right], r \leqslant n$, any solution $y(x, s)$ of $(2.5)$ takes the form

$$
y(x, s)=A_{r}^{(n)}(s) \exp \left(\alpha_{r} s x\right)+B_{r}^{(n)}(s) \exp \left(\beta_{r} s x\right) .
$$

When $r=n+1,(2.29)$ is then valid throughout $\left[a_{n}, \infty\right)$, and condition (2.7) is satisfied if we choose $A_{n+1}^{(n)}=0$ and $B_{n+1}^{(n)}=1$. We denote the solution so obtained by $\phi_{n}(x, s)$. For this solution we have (cf. [4, (3.7)])

$$
\begin{aligned}
& A_{n+2-k}^{(n)}(s)=\sum_{i=1}^{2^{k-1}} C_{k, i}^{(n)} \exp \left(\eta_{k, i}^{(n)} s\right), \\
& B_{n+2-k}^{(n)}(s)=\sum_{i=1}^{2^{k-1}} D_{k, i}^{(n)} \exp \left(\nu_{k, i}^{(n)} s\right)
\end{aligned}
$$

for certain constants $C_{k, i}^{(n)}, D_{k, i}^{(n)}, \eta_{k, i}^{(n)}$, and $\nu_{k, i}^{(n)}$, and for $1 \leqslant k \leqslant n+1$. Set $C_{1,1}^{(n)}=0$, $D_{1,1}^{(n)}=1$, and $\eta_{1,1}^{(n)}=\nu_{1,1}^{(n)}=0$. The condition that $s$ be an eigenvalue is now $\phi_{n}(0, s)$ $=0$, or

$$
A_{1}^{(n)}(s)+B_{1}^{(n)}(s)=0 .
$$

The remainder of this development closely follows that in $[4, \S 3]$, modulo some minor alterations. In particular, the formulae (3.9)-(3.58) in [4] are all valid in the general case, when $\alpha_{r}^{(n)}, \beta_{n}^{(n)}, a_{r}^{(n)}$ are replaced by $\alpha_{r}, \beta_{r}, a_{r}$, respectively, $d_{n}$ replaces $p_{n}^{-\theta}$, and $\lambda_{n}$ replaces $p_{n}$. In all cases the terms $p_{n}^{\theta+s}$ in [4] become $d_{n}^{-1} \lambda_{n}^{s}$ (e.g. in (3.45)-(3.58)). Thus, from the continuity of $\phi_{n}(x, s)$ and $\phi_{n}^{\prime}(x, s)$ at $a_{r}$ we have (cf. $[4,(3.11)-(3.15)])$ that, if

$$
\begin{aligned}
& A_{n+1-k}^{(n)}(s)=\sum_{i=1}^{2^{k}} C_{k+1, i}^{(n)} \exp \left(\eta_{k+1, i}^{(n)} s\right), \\
& B_{n+1-k}^{(n)}(s)=\sum_{i=1}^{2^{k}} D_{k+1, i}^{(n)} \exp \left(\nu_{k+1, i}^{(n)} s\right),
\end{aligned}
$$

then the numbers $C_{k, i}^{(n)}, D_{k, i}^{(n)}, \eta_{k, i}^{(n)}$, and $\nu_{k, i}^{(n)}, 1 \leqslant k \leqslant n$, are generated recursively by the formulae

$$
\begin{aligned}
C_{k+1, i}^{(n)} & =\frac{\alpha_{n+2-k}-\beta_{n+1-k}}{\alpha_{n+1-k}-\beta_{n+1-k}} C_{k, i}^{(n)}, \quad 1 \leqslant i \leqslant 2^{k-1}, \\
& =\frac{\beta_{n+2-k}-\beta_{n+1-k}}{\alpha_{n+1-k}-\beta_{n+1-k}} D_{k, i-2^{k-1}}^{(n)}, \quad 1+2^{k-1} \leqslant i \leqslant 2^{k},
\end{aligned}
$$


(2.36)

$$
\begin{aligned}
D_{k+1, i}^{(n)} & =\frac{\alpha_{n+1-k}-\alpha_{n+2-k}}{\alpha_{n+1-k}-\beta_{n+1-k}} C_{k, i}^{(n)}, \quad 1 \leqslant i \leqslant 2^{k-1}, \\
& =\frac{\alpha_{n+1-k}-\beta_{n+2-k}}{\alpha_{n+1-k}-\beta_{n+1-k}} D_{k, i-2^{k-1}}^{(n)}, \quad 1+2^{k-1} \leqslant i \leqslant 2^{k},
\end{aligned}
$$

(2.37) $\eta_{k+1, i}^{(n)}=\eta_{k, i}^{(n)}+\left(\alpha_{n+2-k}-\alpha_{n+1-k}\right) a_{n+1-k}, \quad 1 \leqslant i \leqslant 2^{k-1}$,

$$
=\nu_{k, i-2^{k-1}}^{(n)}+\left(\beta_{n+2-k}-\alpha_{n+1-k}\right) a_{n+1-k}, \quad 1+2^{k-1} \leqslant i \leqslant 2^{k},
$$

(2.38) $\nu_{k+1, i}^{(n)}=\eta_{k, i}^{(n)}+\left(\alpha_{n+2-k}-\beta_{n+1-k}\right) a_{n+1-k}, \quad 1 \leqslant i \leqslant 2^{k-1}$,

$$
=v_{k, i-2^{k-1}}^{(n)}+\left(\beta_{n+2-k}-\beta_{n+1-k}\right) a_{n+1-k}, \quad 1+2^{k-1} \leqslant i \leqslant 2^{k} .
$$

Using Lemma 2.5, we see that (2.35)-(2.36) become

$$
\begin{aligned}
C_{k+1, i}^{(n)}= & \left(1+\delta_{n+1-k}\right) d_{n+2-k} \cdot \frac{w_{n+1-k}-w_{n-k}}{w_{n+2-k}-w_{n+1-k}} C_{k, i}^{(n)}, \quad 1 \leqslant i \leqslant 2^{k-1}, \\
= & \frac{w_{n+1-k}-w_{n-k}}{w_{n+1-k}} D_{k, i-2^{k-1}}^{(n)}, \quad 1+2^{k-1} \leqslant i \leqslant 2^{k}, \\
D_{k+1, i}^{(n)} & =d_{n+2-k} \cdot \frac{w_{n-k}}{w_{n+2-k}-w_{n+1-k}} C_{k, i}^{(n)}, \quad 1 \leqslant i \leqslant 2^{k-1}, \\
& =\frac{w_{n-k}}{w_{n-k+1}} D_{k, i-2^{k-1}}^{(n)}, \quad 1+2^{k-1} \leqslant i \leqslant 2^{k} .
\end{aligned}
$$

We also have

LEMMA 2.7. (i) For $1 \leqslant k \leqslant n, 1 \leqslant i \leqslant 2^{k}$,

$$
\nu_{k+1, i}^{(n)}=\eta_{k+1, i}^{(n)}+\left(\alpha_{n+1-k}-\beta_{n+1-k}\right) a_{n+1-k} .
$$

(ii) For $k \geqslant 2,1 \leqslant i \leqslant 2^{k-2}$,

$$
\begin{aligned}
& \eta_{k+1,4 i-2}^{(n)}=\eta_{k+1,4 i}^{(n)}-\ln \left(\lambda_{n}\right), \\
& \nu_{k+1,4 i-2}^{(n)}=\nu_{k+1,4 i}^{(n)}-\ln \left(\lambda_{n}\right) .
\end{aligned}
$$

(iii) For $k \geqslant 2,1 \leqslant i \leqslant 2^{k-2}$,

$$
\begin{aligned}
& \eta_{k+1,4 i}^{(n)}-\eta_{k+1,2^{k}}^{(n)}=\eta_{k, 2 i}^{(n-1)}-\eta_{k, 2^{k-1}}^{(n-1)}, \\
& \nu_{k+1,4 i}^{(n)}-\nu_{k+1,2^{k}}^{(n)}=\nu_{k, 2 i}^{(n-1)}-\nu_{k, 2^{k-1}}^{(n-1)} .
\end{aligned}
$$

This is essentially [4, Lemma 3.1].

We next derive generating formulae for $A_{r}^{(n)}$ and $B_{r}^{(n)}$ analogous to that given in Lemma 2.2 for $w_{n}^{(r)}$.

Define $E_{k, i}^{(n)}$ and $F_{k, i}^{(n)}$ by

$$
\begin{gathered}
C_{k, i}^{(n)}=\frac{w_{n-k+2}-w_{n-k+1}}{w_{n}} E_{k, i}^{(n)}, \\
D_{k, i}^{(n)}=\frac{w_{n-k+1}}{w_{n}} F_{k, i}^{(n)} .
\end{gathered}
$$


From (2.39)-(2.40),

$$
\begin{gathered}
E_{k+1, i}^{(n)}=\left(1+\delta_{n+1-k}\right) d_{n+2-k} E_{k, i}^{(n)}, \quad 1 \leqslant i \leqslant 2^{k-1}, \\
=F_{k, i-2^{k-1}}^{(n)}, \quad 1+2^{k-1} \leqslant i \leqslant 2^{k}, \\
F_{k+1, i}^{(n)}=d_{n+2-k} E_{k, i}^{(n)}, \quad 1 \leqslant i \leqslant 2^{k-1}, \\
=F_{k, i-2^{k-1}}^{(n)}, \quad 1+2^{k-1} \leqslant i \leqslant 2^{k} .
\end{gathered}
$$

Also, analogous to [4, Lemma 3.2] we have

LEMMA 2.8. (i) $E_{k, i}^{(n)}=F_{k, i}^{(n)}=0$ if $i$ is odd.

(ii) For $k \geqslant 2$ and $1 \leqslant i \leqslant 2^{k-2}$,

$$
\begin{aligned}
& E_{k+1,4 i}^{(n)}=E_{k, 2 i}^{(n-1)}, \\
& F_{k+1,4 i}^{(n)}=F_{k, 2 i}^{(n-1)} .
\end{aligned}
$$

(iii) For $k \geqslant 4$ and $1 \leqslant i \leqslant 2^{k-4}$,

$$
\begin{gathered}
E_{k, 8 i-2}^{(n)}=d_{n} E_{k, 8 i}^{(n)}, \\
E_{k, 8 i-6}^{(n)}=\left(1+\delta_{n-1}\right) d_{n} E_{k, 8 i-4}^{(n)}, \\
F_{k, 8 i-2}^{(n)}=d_{n} F_{k, 8 i}^{(n)}, \\
F_{k, 8 i-6}^{(n)}=\left(1+\delta_{n-1}\right) d_{n} F_{k, 8 i-4}^{(n)} .
\end{gathered}
$$

Finally, define $L_{r}^{(n)}(s)$ and $M_{r}^{(n)}(s)$ by

$$
\begin{gathered}
A_{r}^{(n)}(s)=\exp \left[s \eta_{n+2-r, 2^{n+1-r}}^{(n)}\right] \cdot \frac{w_{r}-w_{r-1}}{w_{n}} L_{r}^{(n)}(s), \\
B_{r}^{(n)}(s)=\exp \left[s \nu_{n+2-r, 2^{n+1-r}}^{(n)}\right] \cdot \frac{w_{r-1}}{w_{n}} M_{r}^{(n)}(s) .
\end{gathered}
$$

It follows from (2.30)-(2.31), (2.46)-(2.47), and (2.56)-(2.57) that

$$
\begin{aligned}
& L_{r}^{(n)}(s)=\sum_{i=1}^{2^{n+1-r}} E_{n+2-r, i}^{(n)} \exp \left[s\left\{\eta_{n+2-r, i}^{(n)}-\eta_{n+2-r, 2^{n+1-r}}^{(n)}\right\}\right], \\
& M_{r}^{(n)}(s)=\sum_{i=1}^{2^{n+1-r}} F_{n+2-r, i}^{(n)} \exp \left[s\left\{\nu_{n+2-r, i}^{(n)}-\nu_{n+2-r, 2^{n+1-r}}^{(n)}\right\}\right],
\end{aligned}
$$

and, corresponding to [4, Lemma 3.3], we have

LEMMA 2.9. (i)

$$
L_{r}^{(r)}(s)=1, \quad L_{r}^{(r+1)}(s)=1+\left(1+\delta_{r}\right) d_{r+1} / \lambda_{r+1}^{s},
$$

and, for $n \geqslant r+2$,

$$
L_{r}^{(n)}(s)=\left(1+\frac{d_{n}}{\lambda_{n}^{s}}\right) L_{r}^{(n-1)}(s)+\frac{\delta_{n-1} d_{n}}{\lambda_{n}^{s}}\left[L_{r}^{(n-1)}(s)-L_{r}^{(n-2)}(s)\right] .
$$

$$
M_{r}^{(r)}(s)=1, \quad M_{r}^{(r+1)}(s)=1+d_{r+1} / \lambda_{r+1}^{s},
$$


and for $n \geqslant r+2$,

$$
M_{r}^{(n)}(s)=\left(1+\frac{d_{n}}{\lambda_{n}^{s}}\right) M_{r}^{(n-1)}(s)+\frac{\delta_{n-1} d_{n}}{\lambda_{n}^{s}}\left[M_{r}^{(n-1)}(s)-M_{r}^{(n-2)}(s)\right] .
$$

Proceeding now as in the derivation of Lemma 2.3 (cf. [4, (3.47)-(3.58)]), we define functions $H_{r}^{(n)}(s), K_{r}^{(n)}(s), n \geqslant r+1$, by $H_{r}^{(r+1)}(s)=K_{r}^{(r+1)}(s)=1$ and, for $n \geqslant r+2$,

(2.64)

$$
H_{r}^{(n)}(s)=\frac{d_{n}^{-1} \lambda_{n}^{s}\left[L_{r}^{(n)}(s)-L_{r}^{(n-1)}(s)\right]}{\left(1+\left(1+\delta_{r}\right) d_{r+1} / \lambda_{r+1}^{s}\right)\left(1+d_{r+2} / \lambda_{r+2}^{s}\right) \cdots\left(1+d_{n-1} / \lambda_{n-1}^{s}\right)},
$$

$$
K_{r}^{(n)}(s)=\frac{d_{n}^{-1} \lambda_{n}^{s}\left[M_{r}^{(n)}(s)-M_{r}^{(n-1)}(s)\right]}{\left(1+d_{r+1} / \lambda_{r+1}^{s}\right)\left(1+d_{r+2} / \lambda_{r+2}^{s}\right) \cdots\left(1+d_{n-1} / \lambda_{n-1}^{s}\right)} .
$$

Define also

$$
\begin{gathered}
U_{r}^{(n)}(s)=\left(1+\frac{\left(1+\delta_{r}\right) d_{r+1}}{\lambda_{r+1}^{s}}\right)\left(1+\frac{d_{r+2}}{\lambda_{r+2}^{s}}\right) \cdots\left(1+\frac{d_{n}}{\lambda_{n}^{s}}\right), \\
V_{r}^{(n)}(s)=\prod_{j=r+1}^{n}\left(1+\frac{d_{j}}{\lambda_{j}^{s}}\right) .
\end{gathered}
$$

Then, by following the derivation of $[4,(3.53)-(3.54)]$, we obtain

$$
\begin{aligned}
L_{r}^{(n)}(s)=U_{r}^{(n)}(s)\left[1+\delta_{r+1} /(1+\right. & \left.\lambda_{r+1}^{s} /\left(1+\delta_{r}\right) d_{r+1}\right)\left(1+\lambda_{r+2}^{s} / d_{r+2}\right) \\
& \left.+\sum_{j=r+2}^{n-1} \frac{\delta_{j} H_{r}^{(j)}(s)}{\left(1+\lambda_{j}^{s} / d_{j}\right)\left(1+\lambda_{j+1}^{s} / d_{j+1}\right)}\right],
\end{aligned}
$$

where

$$
H_{r}^{(r+2)}(s)=1+\delta_{r+1}\left(1+\lambda_{r+1}^{s}\left(1+\delta_{r}\right)^{-1} d_{r+1}^{-1}\right)^{-1}
$$

and, for $n \geqslant r+3$,

$$
\begin{aligned}
H_{r}^{(n)}(s)= & 1+\frac{\delta_{r+1}}{\left(1+\lambda_{r+1}^{s} /\left(1+\delta_{r}\right) d_{r+1}\right)\left(1+\lambda_{r+2}^{s} / d_{r+2}\right)} \\
& +\sum_{j=r+2}^{n-2} \frac{\delta_{j} H_{r}^{(j)}(s)}{\left(1+\lambda_{j}^{s} / d_{j}\right)\left(1+\lambda_{j+1}^{s} / d_{j+1}\right)}+\frac{\delta_{n-1} H_{r}^{(n-1)}(s)}{\left(1+\lambda_{n-1}^{s} / d_{n-1}\right)}
\end{aligned}
$$

Likewise, analogous to [4, (3.57)-(3.58)] we have

$$
M_{r}^{(n)}(s)=V_{r}^{(n)}(s)\left[1+\sum_{j=r+1}^{n-1} \frac{\delta_{j} K_{r}^{(j)}(s)}{\left(1+\lambda_{j}^{s} / d_{j}\right)\left(1+\lambda_{j+1}^{s} / d_{j+1}\right)}\right]
$$

where

$$
K_{r}^{(r+2)}(s)=1+\delta_{r+1}\left(1+\lambda_{r+1}^{s} d_{r+1}^{-1}\right)^{-1}
$$


and, for $n \geqslant r+3$,

(2.71) $K_{r}^{(n)}(s)=1+\sum_{j=r+1}^{n-2} \frac{\delta_{j} K_{r}^{(j)}(s)}{\left(1+\lambda_{j}^{s} / d_{j}\right)\left(1+\lambda_{j+1}^{s} / d_{j+1}\right)}+\frac{\delta_{n-1} K_{r}^{(n-1)}(s)}{\left(1+\lambda_{n-1}^{s} / d_{n-1}\right)}$.

Thus, on noting that (cf. [4, (4.23)-(4.24)])

$$
\nu_{n+2-r .2^{n+1-r}}^{(n)}=\sum_{k=r}^{n}\left(\beta_{k+1}-\beta_{k}\right) a_{k},
$$

and

$$
\eta_{n+2-r, 2^{n+1-r}}^{(n)}=\sum_{k=r}^{n}\left(\beta_{k+1}-\beta_{k}\right) a_{k}-\ln \left(\lambda_{r}\right)
$$

we obtain from (2.56)-(2.57), (2.68), and (2.70),

$$
A_{r}^{(n)}(s)=\lambda_{r}^{-s} \exp \left[s \sum_{k=r}^{n}\left(\beta_{k+1}-\beta_{k}\right) a_{k}\right] \cdot \frac{w_{r}-w_{r-1}}{w_{n}} U_{r}^{(n)}(s) \Psi_{r}^{(n)}(s),
$$

and

$$
B_{r}^{(n)}(s)=\exp \left[s \sum_{k=r}^{n}\left(\beta_{k+1}-\beta_{k}\right) a_{k}\right] \frac{w_{r-1}}{w_{n}} V_{r}^{(n)}(s) \Phi_{r}^{(n)}(s)
$$

where

$$
\begin{aligned}
\Psi_{r}^{(n)}(s)= & L_{r}^{(n)}(s)\left[U_{r}^{(n)}(s)\right]^{-1} \\
= & 1+\frac{\delta_{r+1}}{\left(1+\lambda_{r+1}^{s} /\left(1+\delta_{r}\right) d_{r+1}\right)\left(1+\lambda_{r+2}^{s} / d_{r+2}\right)} \\
& +\sum_{j=r+2}^{n-1} \frac{\delta_{j} H_{r}^{(j)}(s)}{\left(1+\lambda_{j}^{s} / d_{j}\right)\left(1+\lambda_{j+1}^{s} / d_{j+1}\right)},
\end{aligned}
$$

and

(2.77)

$$
\Phi_{r}^{(n)}(s)=M_{r}^{(n)}(s)\left[V_{r}^{(n)}(s)\right]^{-1}=1+\sum_{j=r+1}^{n-1} \frac{\delta_{j} K_{r}^{(j)}(s)}{\left(1+\lambda_{j}^{s} / d_{j}\right)\left(1+\lambda_{j+1}^{s} / d_{j+1}\right)} .
$$

The solution $\phi_{n}(x, s)$ of (2.5) satisfying (2.7) is now given explicitly for $x$ in $\left[a_{r-1}, a_{r}\right], 1 \leqslant r \leqslant n$, by

$$
\phi_{n}(x, s ; \delta)=A_{r}^{(n)}(s) e^{\alpha_{r} s x}+B_{r}^{(n)}(s) e^{\beta_{r} s x}=\prod_{j=1}^{n}\left(1+\frac{d_{j}}{\lambda_{j}^{s}}\right) \chi_{n}(x, s ; \delta),
$$

where

$$
\chi_{n}(x, s ; \boldsymbol{\delta})=\tilde{A}_{r}^{(n)}(s ; \boldsymbol{\delta}) e^{\alpha_{r} s x}+\tilde{B}_{r}^{(n)}(s ; \delta) e^{\beta_{r} s x}
$$

and

$$
\begin{aligned}
& \tilde{A}_{r}^{(n)}(s ; \delta)= {\left[\prod_{j=1}^{r+1}\left(1+\frac{d_{j}}{\lambda_{j}^{s}}\right)\right]^{-1}\left(1+\frac{\left(1+\delta_{r}\right) d_{r+1}}{\lambda_{r+1}^{s}}\right) } \\
& \cdot \lambda_{r}^{-s} \exp \left[s \sum_{k=r}^{n}\left(\beta_{k+1}-\beta_{k}\right) a_{k}\right] \frac{w_{r}-w_{r-1}}{w_{n}} \Phi_{r}^{(n)}(s),
\end{aligned}
$$




$$
\tilde{B}_{r}^{(n)}(s ; \delta)=\left[\prod_{j=1}^{r}\left(1+\frac{d_{j}}{\lambda_{j}^{s}}\right)\right]^{-1} \exp \left[s \sum_{k=r}^{n}\left(\beta_{k+1}-\beta_{k}\right) a_{k}\right] \cdot \frac{w_{r-1}}{w_{n}} \cdot \Psi_{r}^{(n)}(s) .
$$

Note that as $\delta \rightarrow \mathbf{0}, w_{r} \rightarrow \prod_{j=1}^{r}\left(1+d_{j}\right)$, from Lemma 2.3(i) and, from (2.25), (2.26), and (2.28),

$$
\begin{aligned}
\lim _{\boldsymbol{\delta} \rightarrow 0}\left(\beta_{k+1}-\beta_{k}\right) a_{k} & =\lim _{\delta \rightarrow 0}\left(\beta_{k+1}-\beta_{k}\right)\left(a_{k}-a_{k-1}\right) \\
& =\lim _{\delta \rightarrow 0} \ln \left(\lambda_{k}\right) \cdot \frac{\left(w_{k}-w_{k-1}\right)}{w_{k}}=\frac{\ln \left(\lambda_{k}\right) \cdot d_{k}}{1+d_{k}} .
\end{aligned}
$$

Consequently,

$$
\begin{aligned}
\chi_{n}(0, s ; \mathbf{0}) & =\tilde{A}_{1}^{(n)}(s ; \mathbf{0})+\tilde{B}_{1}^{(n)}(s ; \mathbf{0}) \\
& =w_{n}^{-1} \exp \left[s \sum_{k=1}^{n} \frac{\ln \left(\lambda_{k}\right) \cdot d_{k}}{1+d_{k}}\right] \cdot\left(1+\frac{d_{1}}{\lambda_{1}^{s}}\right)^{-1} \cdot\left\{\lambda_{1}^{-s}\left(w_{1}-w_{0}\right)+1\right\} \\
& =w_{n}^{-1} \exp \left[s \sum_{k=1}^{n} \frac{\ln \left(\lambda_{k}\right) \cdot d_{k}}{1+d_{k}}\right] \\
& \neq 0,
\end{aligned}
$$

which establishes (2.11).

In the next section we show that the existence of the solutions $\phi_{n}(x, s)$ of the equations (2.5), $n \geqslant 1$, may be used to characterize which Dirichlet series have Euler products of the form $\Pi\left(1+d_{n} p_{n}^{-s}\right)$. In this vein it is worth noting that, for a fixed $\delta$, the set of solutions $\phi_{n}(x, s)$ together with their differential equations (2.5) are uniquely associated with (1.3) in the sense that if the sequences $\left\{\alpha_{n}\right\}_{n \geqslant 1},\left\{\beta_{n}\right\}_{n \geqslant 1}$, and $\left\{a_{n}\right\}_{n \geqslant 0}$ (with $\alpha_{1}=0, \beta_{1}=-1, a_{0}=0$ ) defining the differential equation are given and satisfy $\alpha_{n}-\alpha_{n+1}>0$ and $\alpha_{n}-\beta_{n}>0$ for all $n \geqslant 1$, then the original Euler product (1.3) can be recovered as follows. Define (cf. Lemma 2.5(i)) $w_{r}, r \geqslant 0$, by $w_{0}=1$ and

$$
\frac{w_{r}}{w_{r-1}}=\frac{\alpha_{r}-\beta_{r}}{\alpha_{r}-\beta_{r+1}}, \quad r \geqslant 1
$$

then define $d_{r}, \delta_{r}, \lambda_{r}, r \geqslant 1$, by (cf. Lemma 2.6(i), Lemma 2.5(iii)-(iv), and (2.28))

$$
\begin{gathered}
d_{1}=-1 / \beta_{2}-1, \\
d_{r+1}=\frac{\alpha_{r}-\alpha_{r+1}}{\alpha_{r}-\beta_{r}} \cdot \frac{w_{r+1}-w_{r}}{w_{r-1}}, \quad r \geqslant 1, \\
\left(1+\delta_{r}\right)=d_{r+1}^{-1} \frac{\alpha_{r+1}-\beta_{r}}{\alpha_{r}-\beta_{r}} \cdot \frac{w_{r+1}-w_{r}}{w_{r}-w_{r-1}}, \quad r \geqslant 1, \\
\ln \left(\lambda_{r}\right)=\left(a_{r}-a_{r-1}\right)\left(\alpha_{r}-\beta_{r}\right) .
\end{gathered}
$$

Notice that $\beta_{2} \neq 0$ as $\beta_{2}<\alpha_{2}<\alpha_{1}=0$, and

$$
w_{0}=1, \quad w_{1}=-1 / \beta_{2}=1+d_{1} \text {. }
$$


From (2.83)-(2.84) we have, for $r \geqslant 1$,

$$
\begin{aligned}
w_{r-1} d_{r+1} & =\left(w_{r+1}-w_{r}\right) \frac{\alpha_{r}-\alpha_{r+1}}{\alpha_{r}-\beta_{r}}=\left(w_{r+1}-w_{r}\right)\left\{1-\frac{\alpha_{r+1}-\beta_{r}}{\alpha_{r}-\beta_{r}}\right\} \\
& =\left(w_{r+1}-w_{r}\right)-\left(1+\delta_{r}\right) d_{r+1}\left(w_{r}-w_{r-1}\right),
\end{aligned}
$$

i.e.

$$
w_{r+1}=\left(1+d_{r+1}\right) w_{r}+\delta_{r} d_{r+1}\left(w_{r}-w_{r-1}\right) \text {. }
$$

Also, using $\left\{d_{n}\right\}$ and $\left\{\delta_{n}\right\}$ defined above, define $\varepsilon(n)$ by the formulae (2.12)-(2.13) and set

$$
\tilde{w}_{n}=\varepsilon(1)+\varepsilon(2)+\cdots+\varepsilon\left(2^{n}\right) .
$$

Then, by the argument of Lemma 2.2(i),

$$
\tilde{w}_{r+1}=\left(1+d_{r+1}\right) \tilde{w}_{r}+\delta_{r} d_{r+1}\left(\tilde{w}_{r}-\tilde{w}_{r-1}\right) \text {. }
$$

As $\tilde{w}_{0}=\varepsilon(1)=1$ and $\tilde{w}_{1}=\varepsilon(1)+\varepsilon(2)=1+d_{1}$, it follows from (2.86)-(2.87) and (2.89) that $w_{r}=\tilde{w}_{r}=\varepsilon(1)+\cdots+\varepsilon\left(2^{n}\right)$. Consequently, all of the previous formulae in this section connecting $w_{r}, d_{r}, \delta_{r}, a_{r}$, etc., are valid for the values of $\lambda_{r}, d_{r}$, and $\delta_{r}$ defined above. In particular, with these values, the Euler product $\Pi\left(1+d_{n} \lambda_{n}^{-s}\right)$ is then uniquely associated with the given differential equations.

3. Characterization of Euler products. In this section we restrict attention to formal Euler products

$$
E(s)=\prod_{n=1}^{\infty}\left(1+\frac{d_{n}}{p_{n}^{s}}\right),
$$

where $\left\{p_{n}\right\}$ denotes the prime numbers, beginning with $p_{1}=2$. The problem considered here is, given a formal Dirichlet series

$$
D(s)=\sum_{n=1}^{\infty} \frac{a(n)}{n^{s}},
$$

where $a(1)=1$, when can it be written in the form (3.1)? Observe that a necessary condition for this to happen is that $a(n)=0$ if $n$ has a repeated prime factor. In this case one can formally rewrite the series (3.2) in the form

$$
D(s)=\sum_{n=1}^{\infty} \frac{a(q(n))}{q(n)^{s}},
$$

where $q(n)$ is defined by (2.2) with $\lambda_{n}=p_{n}$. Furthermore, (3.2)' has the form (3.1) if and only if $a(\cdot)$ is multiplicative in the sense that

$$
a(m n)=a(m) \cdot a(n) \quad \text { whenever }(m, n)=1,
$$

in which case $d_{n}=a\left(p_{n}\right)$ for each $n$. This is perhaps the simplest example in a much wider theory in which the existence of more complicated Euler products may be used to characterize a wide variety of multiplicative properties of the arithmetic function $a(n)$ (see e.g. $[6,8])$.

The characterization that we derive here depends upon the natural product form of the coefficients $C_{k, i}^{(n)}$ and $D_{k, i}^{(n)}$ in $(2.30)-(2.31)$ and the fact that the product 
structure of (1.3) is essentially only needed (via Lemma 2.2) in passing from the formulae (2.35)-(2.36) to the formulae (2.39)-(2.40). We first associate with (3.2)' differential equations of the type (2.5), considered in the last section, by means of the following construction. Assume that

$$
\sum_{i=1}^{\infty}\left|a\left(p_{i}\right)\right|<\infty
$$

and the sequences $\left\{\delta_{i}\right\}$ and $\left\{\kappa_{i}\right\}$ are chosen so that

$$
\begin{gathered}
0<\kappa_{i} \leqslant 1, \quad i \geqslant 0, \\
\delta_{i} a\left(p_{i}\right)>0, \quad i \geqslant 1, \\
\sup \left\{\left|\delta_{i}\right|: i \geqslant 1\right\} \leqslant \delta^{*},
\end{gathered}
$$

where

$$
\delta^{*}=\frac{1}{8}\left[C+\sum_{i=1}^{\infty} \frac{a\left(p_{i}\right) a\left(p_{i+1}\right)}{\left(1+a\left(p_{i}\right)\right)\left(1+a\left(p_{i+1}\right)\right)}\right]^{-1}
$$

and

$$
C=\sup \left\{\left|a\left(p_{i}\right)\left(a\left(p_{i}\right)+1\right)^{-1}\right|: i \geqslant 1\right\} .
$$

Define the sequence of polynomials $\left\{f_{i}(\boldsymbol{\delta}, \boldsymbol{\kappa})\right\}$ in $\boldsymbol{\delta}=\left\{\boldsymbol{\delta}_{i}\right\}_{i \geqslant 1}$ and $\boldsymbol{\kappa}=\left\{\boldsymbol{\kappa}_{i}\right\}_{i \geqslant 0}$ by

$$
f_{1}(\boldsymbol{\delta}, \boldsymbol{\kappa})=1, \quad f_{2}(\boldsymbol{\delta}, \boldsymbol{\kappa})=1+\kappa_{0},
$$

and, for $n \geqslant 1$,

$$
\begin{aligned}
f_{2^{n}+k}(\boldsymbol{\delta}, \boldsymbol{\kappa}) & =\left(1+\kappa_{n}\right) f_{k}(\boldsymbol{\delta}, \boldsymbol{\kappa}), \quad 1 \leqslant k \leqslant 2^{n-1}, \\
& =\left(1+\boldsymbol{\delta}_{n}\right)\left(1+\kappa_{n}\right) f_{k}(\boldsymbol{\delta}, \boldsymbol{\kappa}), \quad 1+2^{n-1} \leqslant k \leqslant 2^{n} .
\end{aligned}
$$

Set $\hat{\varepsilon}(i)=f_{i}(\boldsymbol{\delta}, \boldsymbol{\kappa}) a(q(i))$ and for $n \geqslant 0,0 \leqslant r \leqslant n$, define (cf. (2.14))

$$
\hat{w}_{n}^{(r)}=\sum_{i=0}^{2^{n-r}-1} \hat{\varepsilon}\left(1+i \cdot 2^{r}\right) .
$$

Let $\{\varepsilon(i): i \geqslant 1\}$ be defined by (2.12)-(2.13) with $d_{n}=\left(1+\kappa_{n-1}\right) a\left(p_{n}\right), n \geqslant 1$. If we set

$$
w_{n}=\sum_{i=0}^{2^{n}-1} \varepsilon(1+i)
$$

and $\hat{w}_{n}^{(0)}=\hat{w}_{n}$, then we have

LEMMA 3.1. The arithmetic function $a(n)$ satisfies (3.3) if and only if $\hat{w}_{n}=w_{n}$ for all $n \geqslant 1$ and all $\delta$ and $\kappa$ small enough.

Proof. Observe that from (3.11),

$$
\hat{w}_{n}=\sum_{i=1}^{2^{n}} f_{i}(\boldsymbol{\delta}, \boldsymbol{\kappa}) a(q(i)),
$$


while $w_{n}$ is equal to a similar sum with the factor $a(q(i))$ replaced by the product $\Pi a(p)$, where $p$ runs through all prime factors of $q(i)$. Thus the result follows easily if, for each fixed $n$, the polynomials $f_{i}(\delta, \kappa), 1 \leqslant i \leqslant 2^{n}$, are linearly independent. We prove this by induction. From (3.10) it is clear that $\left\{f_{1}, f_{2}\right\}$ is independent. Assume that $\left\{f_{i}: 1 \leqslant i \leqslant 2^{n-1}\right\}$ is independent and let $C_{i}, 1 \leqslant i \leqslant 2^{n}$, be constants such that

$$
\sum_{i=1}^{2^{n}} C_{i} f_{i}(\boldsymbol{\delta}, \boldsymbol{\kappa})=0,
$$

i.e., by (3.10), for fixed $\kappa_{n-1}$ and $\delta_{n-1}$,

$$
\begin{aligned}
& \sum_{i=1}^{2^{n-1}} C_{i} f_{i}(\boldsymbol{\delta}, \boldsymbol{\kappa})+\left(1+\kappa_{n-1}\right) \sum_{i=1}^{2^{n-2}} C_{i+2^{n-1}} f_{i}(\boldsymbol{\delta}, \boldsymbol{\kappa}) \\
& \quad+\left(1+\kappa_{n-1}\right)\left(1+\delta_{n-1}\right) \sum_{i=1+2^{n-2}}^{2^{n-1}} C_{i+2^{n-1}} f_{i}(\boldsymbol{\delta}, \boldsymbol{\kappa})=0 .
\end{aligned}
$$

Consequently, by the induction assumption,

$$
\begin{aligned}
& C_{i}+\left(1+\kappa_{n-1}\right) C_{i+2^{n-1}}=0, \quad 1 \leqslant i \leqslant 2^{n-2}, \\
& C_{i}+\left(1+\kappa_{n-1}\right)\left(1+\delta_{n-1}\right) C_{i+2^{n-1}}=0, \quad 1+2^{n-2} \leqslant i \leqslant 2^{n-1},
\end{aligned}
$$

for all $\kappa_{n-1}$ and $\delta_{n-1}$. From (3.13) we have $C_{i}=0,1+2^{n-1} \leqslant i \leqslant 2^{n-2}+2^{n-1}$, and from (3.14), $C_{i}=0,1+2^{n-2}+2^{n-1} \leqslant i \leqslant 2^{n}$. Finally, as $C_{i}=0,1+2^{n-1} \leqslant i$ $\leqslant 2^{n}$, (3.13)-(3.14) also give $C_{i}=0,1 \leqslant i \leqslant 2^{n-1}$, as required.

Next, define

$$
\begin{gathered}
\hat{\alpha}_{1}=0, \quad \hat{\beta}_{1}=-1, \\
\hat{\alpha}_{r}=-\left(\hat{w}_{r}^{(1)}-\hat{w}_{r-1}^{(1)}\right) /\left(\hat{w}_{r}-\hat{w}_{r-1}\right), \quad r \geqslant 1, \\
\hat{\beta}_{r}=-\hat{w}_{r-1}^{(1)} / \hat{w}_{r-1}, \quad r \geqslant 1,
\end{gathered}
$$

and

$$
\hat{b}_{r}=\hat{\alpha}_{r}+\hat{\beta}_{r}, \quad \hat{c}_{r}=\hat{\alpha}_{r} \hat{\beta}_{r} .
$$

Finally, assuming for the moment that $\hat{\alpha}_{r}-\hat{\beta}_{r}>0$ for all $r \geqslant 1$, define $\left\{\hat{a}_{r}\right\}_{r \geqslant 0}$ by $\hat{a}_{0}=0$ and

$$
\hat{a}_{r}=\hat{a}_{r-1}+\left(\ln \left(p_{r}\right)\right) /\left(\hat{\alpha}_{r}-\hat{\beta}_{r}\right) .
$$

By using $\hat{a}_{r}, \hat{b}_{r}, \hat{c}_{r}$ in place of $a_{r}, b_{r}, c_{r}$ in (2.5), one can define a sequence of differential equations

$$
y^{\prime \prime}+s \hat{b}_{n}(x) y^{\prime}+s^{2} \hat{c}_{n}(x) y=0
$$

where the step functions $\hat{b}_{n}(x)$ and $\hat{c}_{n}(x)$ are defined as in (2.9) with $\hat{b}_{n}$ and $\hat{c}_{n}$ replacing $b_{n}$ and $c_{n}$, respectively. By analogy with (2.29)-(2.31) and (2.37)-(2.40) one can also define a sequence of functions $\hat{\phi}_{n}(x, s ; \delta, \kappa)$ by

$$
\begin{aligned}
\hat{\phi}_{n}(x, s) & =\hat{A}_{r}^{(n)}(s) \exp \left(\hat{\alpha}_{r} s x\right)+\hat{B}_{r}^{(n)}(s) \exp \left(\hat{\beta}_{r} s x\right), \quad x \in\left[\hat{a}_{r-1}, \hat{a}_{r}\right), 1 \leqslant r \leqslant n, \\
& =\exp \left(\hat{\beta}_{n+1} s x\right), \quad x \geqslant \hat{a}_{n},
\end{aligned}
$$


where $\hat{A}_{r}^{(n)}(s)$ and $\hat{B}_{r}^{(n)}(s)$ are given by

$$
\begin{aligned}
& \hat{A}_{n+2-k}^{(n)}(s)=\sum_{i=1}^{2^{k-1}} \hat{C}_{k, i}^{(n)} \exp \left(\hat{\eta}_{k, i}^{(n)} s\right), \\
& \hat{B}_{n+2-k}^{(n)}(s)=\sum_{i=1}^{2^{k-1}} \hat{D}_{k, i}^{(n)} \exp \left(\hat{\nu}_{k, i}^{(n)} s\right),
\end{aligned}
$$

and $\hat{C}_{k, i}^{(n)}, \hat{D}_{k, i}^{(n)}, \hat{\eta}_{k, i}^{(n)}, \hat{\nu}_{k, i}^{(n)}$ are given by $\hat{C}_{1,1}^{(n)}=0, \hat{D}_{1,1}^{(n)}=1, \hat{\eta}_{1,1}^{(n)}=\hat{\nu}_{1,1}^{(n)}=0$, and (cf. (2.37)-(2.40))

$$
\begin{aligned}
\hat{C}_{k+1, i}^{(n)}= & \left(1+\delta_{n+1-k}\right)\left(1+\kappa_{n+1-k}\right) a\left(p_{n+2-k}\right) \\
& \cdot \frac{\hat{w}_{n+1-k}-\hat{w}_{n-k}}{\hat{w}_{n+2-k}-\hat{w}_{n+1-k}} \hat{C}_{k, i}^{(n)}, \quad 1 \leqslant i \leqslant 2^{k-1}, \\
= & \frac{\hat{w}_{n+1-k}-\hat{w}_{n-k}}{\hat{w}_{n+1-k}} \hat{D}_{k, i-2^{k-1}}^{(n)}, \quad 1+2^{k-1} \leqslant i \leqslant 2^{k},
\end{aligned}
$$

$$
\begin{aligned}
\hat{D}_{k+1, i}^{(n)} & =\left(1+\kappa_{n+1-k}\right) a\left(p_{n+2-k}\right) \frac{\hat{w}_{n-k}}{\hat{w}_{n+2-k}-\hat{w}_{n+1-k}} \hat{C}_{k, i}^{(n)}, \quad 1 \leqslant i \leqslant 2^{k-1}, \\
& =\frac{\hat{w}_{n-k}}{\hat{w}_{n-k+1}} \hat{D}_{k, i-2^{k-1}}^{(n)}, \quad 1+2^{k-1} \leqslant i \leqslant 2^{k}
\end{aligned}
$$

$$
\begin{aligned}
\hat{\eta}_{k+1, i}^{(n)} & =\hat{\eta}_{k, i}^{(n)}+\left(\hat{\alpha}_{n+2-k}-\hat{\alpha}_{n+1-k}\right) \hat{a}_{n+1-k}, \quad 1 \leqslant i \leqslant 2^{k-1}, \\
& =\hat{\nu}_{k, i-2^{k-1}}^{(n)}+\left(\hat{\beta}_{n+2-k}-\hat{\alpha}_{n+1-k}\right) \hat{a}_{n+1-k}, \quad 2^{k-1}+1 \leqslant i \leqslant 2^{k}, \\
\hat{\nu}_{k+1, i}^{(n)} & =\hat{\eta}_{k, i}^{(n)}+\left(\hat{\alpha}_{n+2-k}-\hat{\beta}_{n+1-k}\right) \hat{a}_{n+1-k}, \quad 1 \leqslant i \leqslant 2^{k-1}, \\
& =\hat{\nu}_{k, i-2^{k-1}}^{(n)}+\left(\hat{\beta}_{n+2-k}-\hat{\beta}_{n+1-k}\right) \hat{a}_{n+1-k}, \quad 2^{k-1}+1 \leqslant i \leqslant 2^{k} .
\end{aligned}
$$

Observe that by the procedure following (2.47), one can show that $\hat{A}_{r}^{(n)}$ and $\hat{B}_{r}^{(n)}$ are given by (2.74)-(2.75) with $\lambda_{r}, d_{r}, \beta_{r}, a_{r}, w_{r}$ being replaced by $p_{r},\left(1+\kappa_{r-1}\right) a\left(p_{r}\right)$, $\hat{\beta}_{r}, \hat{a}_{r}$, and $\hat{w}_{r}$, respectively.

The main result of this section can now be stated.

TheOREM 3.2. The Dirichlet series (3.2)' can be written in the Euler product form (3.1) (with $d_{n}=a\left(p_{n}\right)$ ) if and only if for each $n \geqslant 1$ and $\delta, \kappa$ satisfying (3.5)-(3.7), $\hat{\alpha}_{n}-\hat{\beta}_{n}>0$ and $\hat{\phi}_{n}(x, s ; \delta, \kappa)$ is a solution of the differential equation (3.20). In this case $\hat{\phi}_{n}$ is the unique eigenfunction for the eigenvalue problem consisting of (3.20) and the boundary conditions

$$
y(0, s)=0, \quad y(x, s) \sim \exp \left(\hat{\beta}_{n+1} s x\right) \text { as } x \rightarrow \infty .
$$

Proof. If (3.2) has the form (3.1), the result follows by the construction in $\$ 2$, as we then have $\hat{\varepsilon}(i)=\varepsilon(i), \hat{w}_{n}=w_{n}$, etc. Conversely, if $\hat{\alpha}_{n}-\hat{\beta}_{n}>0$ and $\hat{\phi}_{n}$ satisfies (3.20) for all $n, \delta, \kappa$ as indicated above, it follows from (2.35)-(2.36) that the 
constants $\hat{C}_{k, i}^{(n)}$ and $\hat{D}_{k, i}^{(n)}$ are also generated by the equations

$$
\begin{aligned}
\hat{C}_{k+1, i}^{(n)}= & \frac{\hat{\alpha}_{n+2-k}-\hat{\beta}_{n+1-k}}{\hat{\alpha}_{n+1-k}-\hat{\beta}_{n+1-k}} \cdot \hat{C}_{k, i}^{(n)}, \quad 1 \leqslant i \leqslant 2^{k-1}, \\
= & \frac{\hat{\beta}_{n+2-k}-\hat{\beta}_{n+1-k}}{\hat{\alpha}_{n+1-k}-\hat{\beta}_{n+1-k}} \cdot \hat{D}_{k, i-2^{k-1}}^{(n)}, \quad 1+2^{k-1} \leqslant i \leqslant 2^{k}, \\
\hat{D}_{k+1, i}^{(n)}= & \frac{\hat{\alpha}_{n+1-k}-\hat{\alpha}_{n+2-k}}{\hat{\alpha}_{n+1-k}-\hat{\beta}_{n+1-k}} \cdot \hat{C}_{k, i}^{(n)}, \quad 1 \leqslant i \leqslant 2^{k-1}, \\
= & \frac{\hat{\alpha}_{n+1-k}-\hat{\beta}_{n+2-k}}{\hat{\alpha}_{n+1-k}-\hat{\beta}_{n+1-k}} \cdot \hat{D}_{k, i-2^{k-1}}^{(n)}, \quad 1+2^{k-1} \leqslant i \leqslant 2^{k} .
\end{aligned}
$$

Consequently, comparing (3.28)-(3.29) with (3.23)-(3.24), we see that, for $r>2$,

$$
\frac{\hat{\alpha}_{r}-\hat{\beta}_{r-1}}{\hat{\alpha}_{r-1}-\hat{\beta}_{r-1}}=\left(1+\delta_{r-1}\right)\left(1+\kappa_{r-1}\right) a\left(p_{r}\right) \frac{\hat{w}_{r-1}-\hat{w}_{r-2}}{\hat{w}_{r}-\hat{w}_{r-1}}
$$

and

$$
\frac{\hat{\alpha}_{r-1}-\hat{\alpha}_{r}}{\hat{\alpha}_{r-1}-\hat{\beta}_{r-1}}=\left(1+\kappa_{r-1}\right) a\left(p_{r}\right) \frac{\hat{w}_{r-2}}{\hat{w}_{r}-\hat{w}_{r-1}} .
$$

Adding (3.30) and (3.31) and rearranging gives

$$
\hat{w}_{r}=\left[1+\left(1+\kappa_{r-1}\right) a\left(p_{r}\right)\right] \hat{w}_{r-1}+\delta_{r-1}\left(1+\kappa_{r-1}\right) a\left(p_{r}\right)\left[\hat{w}_{r-1}-\hat{w}_{r-2}\right]
$$

for $r \geqslant 2$, where $\hat{w}_{0}=1$ and $\hat{w}_{1}=1+\left(1+\kappa_{0}\right) a\left(p_{1}\right)$. From (2.12)-(2.13), (3.12), and Lemma 2.2 with $d_{r}=\left(1+\kappa_{r-1}\right) a\left(p_{r}\right)$, we also have $w_{0}=1, w_{1}=1+$ $\left(1+\kappa_{0}\right) a\left(p_{1}\right)$, and

$$
w_{r}=\left[1+\left(1+\kappa_{r-1}\right) a\left(p_{r}\right)\right] w_{r-1}+\delta_{r-1}\left(1+\kappa_{r-1}\right) a\left(p_{r}\right)\left[w_{r-1}-w_{r-2}\right] .
$$

As $w_{r}$ and $\hat{w}_{r}$ have the same values for $r=0,1$ and satisfy the same generating formula, it follows that $w_{r}=\hat{w}_{r}$ for all $r \geqslant 0$ and, hence, by Lemma 3.1 , the arithmetic function $a(\cdot)$ is multiplicative. This completes the proof.

It seems reasonable to conjecture that a similar characterization should be possible for more general Euler product expressions. For example, one would expect an Euler product of the form

$$
E(s)=\prod_{p \text { prime }}\left(1+\frac{c(p)}{p^{s}}+\frac{d(p)}{p^{2 s}}\right)
$$

to be associated with a third-order differential equation (this would allow the number of terms in $\phi_{n}(0, s)$ to treble each time $n$ increases by one, corresponding to adding one more factor $\left.1+c(p) p^{-s}+d(p) p^{-2 s}\right)$. This more general theory might then provide a useful alternative to the Hecke-Petersson modular form approach. In particular, it may be possible to make use of the natural multiplicative structure provided by the differential equations (analogous to that of the constants $C_{k, i}^{(n)}$ and $D_{k, i}^{(n)}$ above, for example) to determine new multiplication identities satisfied by $c(\cdot)$ and $d(\cdot)$ in (3.33).

4. The limiting differential equation. In this section we investigate some of the consequences arising from letting $n$ tend to infinity in the construction of $\$ 2$.

Henceforth we assume that, in addition to (1.1), (1.2), (2.10), and (2.21),

$$
\sum_{n=1}^{\infty} \ln \left(\lambda_{n}\right) \cdot\left|d_{n}\right|<\infty
$$


the Euler product (1.3) has abscissa of absolute convergence $\sigma_{0}$, i.e.,

$$
\sum_{n=1}^{\infty} \frac{\left|d_{n}\right|}{\lambda_{n}^{\sigma}}<\infty
$$

for $\sigma=\operatorname{Re}(s)>\sigma_{0}$, and

$$
\sum_{n=1}^{\infty}\left(\prod_{k=1}^{n} \frac{\delta_{k} d_{k}}{\lambda_{k}^{\sigma}}\right)<\infty
$$

for $\sigma=\operatorname{Re}(s)>\sigma_{2}$. As a consequence of (1.2), Lemma 2.3, and Lemma 2.4, it follows that for fixed $r$ the numbers $w_{n}^{(r)}$ (defined by (2.14)) tend to a finite positive limit as $n \rightarrow \infty$, which we denote by $w_{\infty}^{(r)}$, i.e.

$$
w_{\infty}^{(r)}=\sum_{k=0}^{\infty} \varepsilon\left(1+k \cdot 2^{r}\right) .
$$

Corresponding to [4, Lemmas 2.3, 2.4(ii)] we have

LEMMA 4.1. (i) For $1 \leqslant n<\infty$ and $1 \leqslant r \leqslant n-1$, set $v_{n}^{(r)}=w_{n}^{(r-1)}-w_{n}^{(r)}$. Then

$$
v_{n}^{(r)}=d_{r}\left[w_{n}^{(r)}+\delta_{r}\left(w_{n}^{(r)}-w_{n}^{(r+1)}\right)\right]
$$

(ii) For $r \geqslant 1$,

$$
w_{\infty}^{(r-1)}-w_{\infty}^{(r)}=d_{r}\left[w_{\infty}^{(r)}+\delta_{r}\left(w_{\infty}^{(r)}-w_{\infty}^{(r+1)}\right)\right] .
$$

(iii) For fixed $r \geqslant 2$ and $1 \leqslant k \leqslant r-1$,

$$
w_{r}^{(k-1)} w_{\infty}^{(k)}-w_{r}^{(k)} w_{\infty}^{(k-1)}=\delta_{k} d_{k}\left[w_{r}^{(k)} w_{\infty}^{(k+1)}-w_{r}^{(k+1)} w_{\infty}^{(k)}\right]
$$

Also, corresponding to [4, (4.4)],

$$
\begin{aligned}
\beta_{r}+\frac{w_{\infty}^{(1)}}{w_{\infty}} & =-\frac{w_{r-1}^{(1)}}{w_{r-1}}+\frac{w_{\infty}^{(1)}}{w_{\infty}}=\frac{w_{r-1} w_{\infty}^{(1)}-w_{\infty} w_{r-1}^{(1)}}{w_{\infty} w_{r-1}} \\
& =\left(\delta_{1} d_{1}\right)\left(\delta_{2} d_{2}\right) \cdots\left(\delta_{r-2} d_{r-2}\right) \cdot \frac{w_{r-1}^{(r-2)} w_{\infty}^{(r-1)}-w_{r-1}^{(r-1)} w_{\infty}^{(r-2)}}{w_{\infty} w_{r-1}} \\
& \quad \text { (by repeated application of Lemma 4.1(iii)) } \\
& =-\left(\prod_{i=1}^{r-2} \delta_{i} d_{i}\right) \cdot \frac{\delta_{r-1} d_{r-1}\left(w_{\infty}^{(r-1)}-w_{\infty}^{(r)}\right)}{w_{\infty} w_{r-1}} \\
& =-\left(\prod_{i=1}^{r-1} \delta_{i}\right) \cdot\left(\prod_{i=1}^{r} d_{i}\right) \cdot \frac{w_{\infty}^{(r)}+\delta_{r}\left(w_{\infty}^{(r)}-w_{\infty}^{(r+1)}\right)}{w_{r-1} w_{\infty}}
\end{aligned}
$$

(by Lemma 4.1(iii) again).

Setting

$$
\gamma=-w_{\infty}^{(1)} / w_{\infty}
$$

we see that, from (1.2), $d_{r} \rightarrow 0$ as $r \rightarrow \infty$ and, from (2.20), $\delta_{r} d_{r}<1$ eventually. Consequently, by (4.5) and (2.25), $\alpha_{r} \rightarrow \gamma$ and $\beta_{r} \rightarrow \gamma$ as $r \rightarrow \infty$. 
Define functions $b(x)$ and $c(x)$ on $[0, \infty)$ by $b(x)=b_{r}$ and $c(x)=c_{r}$ for $x$ in $\left[a_{r-1}, a_{r}\right), r \geqslant 1$, where $b_{r}$ and $c_{r}$ are defined in (2.27). Clearly, for each fixed $x$ in $[0, \infty)$ we have $b_{n}(x) \rightarrow b(x)$ and $c_{n}(x) \rightarrow c(x)$ as $n \rightarrow \infty$. Formally letting $n \rightarrow \infty$ in the differential equation (2.5), we obtain the equation

$$
y^{\prime \prime}-s b(x) y^{\prime}+s^{2} c(x) y=0,
$$

while the boundary condition (2.7) becomes

$$
y(x, s) \sim e^{\gamma s x} \text { as } x \rightarrow \infty .
$$

It is convenient here to transform (4.7)-(4.8) by means of the change of variable $y(x, s)=e^{\gamma s x} z(x, s)$ :

$$
\begin{gathered}
z^{\prime \prime}-s \tilde{b}(x) z^{\prime}+s^{2} \tilde{c}(s) z=0, \\
z(x, s) \sim 1 \text { as } x \rightarrow \infty
\end{gathered}
$$

where $\tilde{b}(x)=b(x)-2 \gamma$ and $\tilde{c}(x)=c(x)-\gamma b(x)+\gamma^{2}$. Observe that on $\left[a_{r-1}, a_{r}\right)$, $\tilde{c}(x)=\tilde{c}_{n}$, where

$$
\begin{aligned}
\tilde{c}_{n} & =\alpha_{n} \beta_{n}-\gamma\left(\alpha_{n}+\beta_{n}\right)+\gamma^{2}=\left(\beta_{n}-\gamma\right)^{2}+\left(\beta_{n}-\gamma\right)\left(\alpha_{n}-\beta_{n}\right) \\
& =\left(\beta_{n}-\gamma\right)\left(\alpha_{n}-\beta_{n}\right)\left[1+\left(\beta_{n}-\gamma\right) /\left(\alpha_{n}-\beta_{n}\right)\right] \\
& \sim\left(\beta_{n}-\gamma\right)\left(\alpha_{n}-\beta_{n}\right) \text { as } n \rightarrow \infty
\end{aligned}
$$

by (2.25), Lemma 2.2(ii), and (4.5). Also, on the same interval $\tilde{b}(x)=\tilde{b}_{n}$, where

$$
\begin{aligned}
\tilde{b}_{n} & =2\left(\beta_{n}-\gamma\right)+\left(\alpha_{n}-\beta_{n}\right)=\left(\alpha_{n}-\beta_{n}\right)\left[1+2\left(\beta_{n}-\gamma\right) /\left(\alpha_{n}-\beta_{n}\right)\right] \\
& \sim \alpha_{n}-\beta_{n} \text { as } n \rightarrow \infty
\end{aligned}
$$

by the same reasoning. Equation (4.9) may be rewritten in the form

$$
\frac{d}{d x}\left[\exp \left(-s \int_{0}^{x} \tilde{b}\right) \frac{d z}{d x}\right]+s^{2} \tilde{c}(x) \exp \left(-s \int_{0}^{x} \tilde{b}\right) \cdot z=0 .
$$

On using the change of independent variable defined by

$$
r(x)=\int_{0}^{x} \tilde{b}(w) d w
$$

with

$$
u(r, s)=z(x(r), s)
$$

we finally obtain

$$
\frac{d}{d r}\left[e^{-s r} \tilde{b}(x(r)) \frac{d u}{d r}\right]+s^{2} \frac{\tilde{c}(x(r))}{\tilde{b}(x(r))} e^{-s r} u(r, s)=0 .
$$

Observe that

$$
\begin{aligned}
r_{n} & \equiv r\left(a_{n}\right)=\sum_{r=1}^{n} \int_{a_{r-1}}^{a_{r}} \tilde{b}(w) d w=\sum_{r=1}^{n}\left(a_{r}-a_{r-1}\right) \tilde{b}_{r} \\
& \sim \sum_{r=1}^{n} \ln \left(\lambda_{r}\right)=\ln \left(\lambda_{1} \lambda_{2} \cdots \lambda_{n}\right)
\end{aligned}
$$

as $n \rightarrow \infty$, by (4.12). 
These differential equations and the associated integral equations (see below) have a number of interesting properties relating to the analytic function $E(s)$.

In the seque! we assume, in addition to (1.1), (1.2), (2.10), (2.21), and (4.1)-(4.3), that

$$
\lambda_{j} \geqslant 1+\mu, \quad j \geqslant 1,
$$

for some $\mu>0$.

We begin with

LEMMA 4.1. If for some $s, \operatorname{Re} s>\sigma_{2}$ (where $\sigma_{2}$ is defined in (4.3)), the function $z(x, s)$ is a solution of (4.9) and satisfies $z(x, s) \rightarrow 1$ as $x \rightarrow \infty$, then

$$
\exp \left(-s \int_{0}^{x} \tilde{b}(w) d w\right) z^{\prime}(x, s) \rightarrow 0 \text { as } x \rightarrow \infty \text {. }
$$

Proof. Observe first that on integrating (4.13) from 0 to $x$, one obtains

$$
z^{\prime}(x, s) \exp \left(-s \int_{0}^{x} \tilde{b}\right)=z^{\prime}(0, s)-s^{2} \int_{0}^{x} \tilde{c}(v) \cdot \exp \left(-s \int_{0}^{v} \tilde{b}\right) \cdot z(v, s) d v .
$$

As a consequence, $z^{\prime}(x, s) \exp \left(-s \int_{0}^{x} \tilde{b}\right)$ approaches a finite limit, $l(s)$, as $x \rightarrow \infty$. This is obvious if $s=0$, while if $s \neq 0$ and $\operatorname{Re} s=\sigma$, we have

$$
\begin{aligned}
\int_{0}^{\infty} & \left|\tilde{c}(v) \exp \left(-s \int_{0}^{l^{\prime}} \tilde{b}\right)\right| d v=\sum_{k=1}^{\infty} \int_{a_{k-1}}^{a_{k}}\left|\tilde{c}(v) \exp \left(-s \int_{0}^{v} \tilde{b}\right)\right| d v \\
= & \sum_{k=1}^{\infty} \exp \left(-\sigma \int_{0}^{a_{k-1}} \tilde{b}\right)\left|\tilde{c}_{k}\right| \int_{a_{k-1}}^{a_{k}} \exp \left(-\sigma\left(v-a_{k-1}\right) \tilde{b}_{k}\right) d v \\
= & \sum_{k=1}^{\infty} \exp \left(-\sigma \sum_{i=1}^{k-1}\left(a_{i}-a_{i-1}\right) \tilde{b}_{i}\right)\left|\tilde{c}_{k}\right|\left(-\sigma \tilde{b}_{k}\right)^{-1} \cdot\left[\exp \left(-\sigma\left(a_{k}-a_{k-1}\right) \tilde{b}_{k}\right)-1\right] \\
= & O(1) \sum_{k=1}^{\infty}\left|\tilde{c}_{k}\right| \tilde{b}_{k}^{-1}\left[\left(\lambda_{1} \cdots \lambda_{k}\right)^{-\sigma}-\left(\lambda_{1} \cdots \lambda_{k-1}\right)^{-\sigma}\right] \\
& \quad\left(\text { as }\left(a_{r}-a_{r-1}\right) \tilde{b}_{r}=\ln \left(\lambda_{r}\right)\left[1+O(1) \cdot d_{r}\right] \text { from }(2.28),(2.25),\right. \text { and (4.5)) } \\
= & O(1) \sum_{k=1}^{\infty} \prod_{i=1}^{k} \frac{\delta_{i} d_{i}}{\lambda_{i}^{\sigma}} \\
< & \infty \quad \text { by }(4.3) .
\end{aligned}
$$

Thus, as $|z|$ is bounded, the right side of (4.19) approaches a limit, as required. We now show that $l(s)=0$ for all $s, \operatorname{Re} s>\sigma_{1}$. Assume $l \neq 0$. There are several cases. If $s=0$ choose $N$ such that $\left|z^{\prime}(x, 0)-l(0)\right|<\varepsilon$ for some $\varepsilon, 0<\varepsilon<|l(0)|$, and $\left|z\left(a_{n}, 0\right)-z\left(a_{n-1}, 0\right)\right|<1$ for all $x, n>N$. Then

$$
\begin{aligned}
1 & >\left|z\left(a_{n}, 0\right)-z\left(a_{n-1}, 0\right)\right|=\left|\int_{a_{n-1}}^{a_{n}} z^{\prime}(x, 0) d x\right| \\
& =\left|\int_{a_{n-1}}^{a_{n}}\left\{\left(z^{\prime}(x, 0)-l(0)\right)+l(0)\right\} d x\right| \\
& \geqslant(|l(0)|-\varepsilon) \cdot\left(a_{n}-a_{n-1}\right) .
\end{aligned}
$$


Since $\left|\delta_{r} d_{r}\right| \leqslant \delta^{*}\left|d_{r}\right| \rightarrow 0$ as $r \rightarrow \infty$, it follows from (2.25) and (2.28) that $a_{n}-a_{n-1}$ $\rightarrow \infty$ as $n \rightarrow \infty$ and, hence, (4.20) provides a contradiction to the assumption that $l(0) \neq 0$. If $s=i t, t \neq 0$, by a similar argument choose $\varepsilon$ so that $0<\varepsilon<2 \pi^{-1} \mid l($ it $) \mid$ and $N$ so that

$$
\begin{gathered}
\left|z\left(x_{2}, s\right)-z\left(x_{1}, s\right)\right|<1, \\
\left|z^{\prime}(x, s) \exp \left(-s \int_{0}^{x} \tilde{b}\right)-l(s)\right|<\varepsilon, \\
\left|2\left(\beta_{n}-\gamma\right) /\left(\alpha_{n}-\beta_{n}\right)\right|<1 / 2
\end{gathered}
$$

for all $x, x_{1}, x_{2}, n>N$. Then for $N \leqslant a_{n-1} \leqslant x_{1}<x_{2} \leqslant a_{n}$ we have

$$
\begin{aligned}
1 & >\left|z\left(x_{2}, s\right)-z\left(x_{1}, s\right)\right| \\
& =\left|\int_{x_{1}}^{x_{2}}\left[\left\{z^{\prime}(x, s) \exp \left(-s \int_{0}^{x} \tilde{b}\right)-l(s)\right\} \cdot \exp \left(s \int_{0}^{x} \tilde{b}\right)+l(s) \exp \left(s \int_{0}^{x} \tilde{b}\right)\right] d x\right| \\
& \geqslant|l(i t)|\left|\int_{x_{1}}^{x_{2}} \exp \left(i t \int_{x_{1}}^{x} \tilde{b}\right) d x\right|-\varepsilon\left(x_{2}-x_{1}\right) \\
& =\frac{|l(i t)|}{|t| \tilde{b}_{n}}\left|\exp \left(i t\left(x_{2}-x_{1}\right) \tilde{b}_{n}\right)-1\right|-\varepsilon\left(x_{2}-x_{1}\right) \\
& =\frac{|l(i t)|}{|t| \tilde{b}_{n}} \cdot 2\left|\sin \left(\frac{1}{2} t\left(x_{2}-x_{1}\right) \tilde{b}_{n}\right)\right|-\varepsilon\left(x_{2}-x_{1}\right) .
\end{aligned}
$$

As

$$
\begin{aligned}
0 & <\frac{1}{2}|t| \ln (1+\mu) \cdot\left(1-\frac{1}{2}\right) \leqslant \frac{1}{2}|t| \ln \left(\lambda_{n}\right)\left[1+\frac{2\left(\beta_{n}-\gamma\right)}{\alpha_{n}-\beta_{n}}\right] \\
& =\frac{1}{2}|t|\left(a_{n}-a_{n-1}\right) \tilde{b}_{n}
\end{aligned}
$$

by (4.18), we can choose $x_{1}^{(n)}=a_{n-1}$ and $x_{2}^{(n)}$ so that

$$
\frac{1}{2}|t|\left(x_{2}^{(n)}-x_{1}^{(n)}\right) \tilde{b}_{n}=\frac{1}{2}|t| \cdot \frac{1}{2} \ln (1+\mu) .
$$

Furthermore by choosing $\mu$, which is independent of $n$, smaller if necessary we can assume that the right side in (4.25) is smaller than $\pi / 2$. Then from the inequality $|\sin \theta|>2 \pi^{-1} \theta(|\theta|<\pi / 2)$ and (4.24) we have that

$$
\begin{aligned}
1 & \geqslant\left(2 \pi^{-1}|l(i t)|-\varepsilon\right)\left(x_{2}^{(n)}-x_{1}^{(n)}\right) \\
& =\left(2 \pi^{-1}|l(i t)|-\varepsilon\right) \cdot \frac{1}{2} \ln (1+\mu) \cdot \tilde{b}_{n}^{-1}
\end{aligned}
$$

by (4.25). As $\tilde{b}_{n} \rightarrow 0$ when $n \rightarrow \infty$, we again have a contradiction. Finally, if $s \neq 0$ and $\sigma=\operatorname{Re}(s) \neq 0$, choose $\varepsilon$ so that $0<\varepsilon<\sigma|l(s)||s|^{-1}$ and $N$ so that (4.21)-(4.23) 
hold for all $x, x_{1}, x_{2}, n>N$. Then, as above, for $N \leqslant a_{n-1} \leqslant x_{1}<x_{2} \leqslant a_{n}$,

$$
\begin{aligned}
1 & \geqslant\left|z\left(x_{2}, s\right)-z\left(x_{1}, s\right)\right| \\
& \geqslant|l(s)|\left|\int_{x_{1}}^{x_{2}} \exp \left(s \int_{0}^{x} \tilde{b}\right) d x\right|-\varepsilon \int_{x_{1}}^{x_{2}} \exp \left(\sigma \int_{0}^{x} \tilde{b}\right) d x \\
& =\exp \left(\sigma \int_{0}^{x_{1}} \tilde{b}\right)\left\{\frac{|l(s)|}{\tilde{b}_{n}|s|}\left|\exp \left(s\left(x_{2}-x_{1}\right) \tilde{b}_{n}\right)-1\right|\right. \\
& \left.\quad-\frac{\varepsilon}{\sigma \tilde{b}_{n}}\left|\exp \left(\sigma\left(x_{2}-x_{1}\right) \tilde{b}_{n}\right)-1\right|\right\} .
\end{aligned}
$$

If $\sigma>0$ (the proof for $\sigma<0$ is similar) it follows that

$$
1 \geqslant \tilde{b}_{n}^{-1} \exp \left(\sigma \int_{0}^{x_{1}} \tilde{b}\right)\left(\frac{|l(s)|}{|s|}-\frac{\varepsilon}{\sigma}\right)\left\{\exp \left(\sigma\left(x_{2}-x_{1}\right) \tilde{b}_{n}\right)-1\right\} .
$$

By a similar argument to that above, for each $n$ large enough we can choose $x_{1}^{(n)}=a_{n-1}$ and $x_{2}^{(n)}$ so that

$$
\tilde{b}_{n}\left(x_{2}^{(n)}-x_{1}^{(n)}\right)=\frac{1}{2} \ln (1+\mu)
$$

Observe also that

$$
\exp \left(\sigma \int_{0}^{a_{n-1}} \tilde{b}\right) / \tilde{b}_{n} \sim \prod_{i=1}^{n-1}\left(\frac{\lambda_{i}^{\sigma}}{\delta_{i} d_{i}}\right)
$$

as $n \rightarrow \infty$, and $\prod_{i=1}^{n-1} \lambda_{i}^{\sigma}\left(\delta_{i} d_{i}\right)^{-1} \rightarrow \infty$ as $n \rightarrow \infty$ by (4.3). Using this information in (4.26), we again obtain a contradiction. This completes the proof.

It is not difficult to see that if $u(r, s)$ is a solution of (4.16) satisfying $u(r, s) \rightarrow 1$ and $e^{-s r} \tilde{b}(x(r)) d u / d r \rightarrow 0$ as $r \rightarrow r(\infty)=r_{\infty}$, then, on integrating (4.16) twice, $u(r, s)$ satisfies the integral equation

$$
u(r, s)=1-s^{2} \int_{r}^{r_{\infty}} \int_{t}^{r_{\infty}} e^{-s(w-t)} \frac{\tilde{c}(x(w))}{\tilde{b}(x(t)) \tilde{b}(x(w))} u(w, s) d w d t
$$

With the aid of Lemma 4.1 this result may be strengthened as follows:

THEOREM 4.2. (i) $A$ locally integrable function $u(r, s), \operatorname{Re}(s)>\sigma_{2}$, is a solution of (4.28) if and only if $u(r, s)$ satisfies the differential equation (4.16) and $u(r, s) \rightarrow 1$ as $r \rightarrow r_{\infty}$.

(ii) The integral equation (4.28) has a unique bounded solution if

$$
\int_{0}^{r_{\infty}} \int_{t}^{r_{\infty}} e^{-\operatorname{Re}(s)(w-t)}\left|\frac{\tilde{c}(x(w))}{\tilde{b}(x(w)) \tilde{b}(x(t))}\right| d w d t<\infty .
$$

Proof. (i) if $u(r, s)$ satisfies (4.16), and $u(r, s) \rightarrow 1$ as $r \rightarrow r_{\infty}$, it follows from Lemma 4.1 that $e^{-s r} \tilde{b}(x(r)) d u / d r \rightarrow 0$ as $r \rightarrow r_{\infty}$ and, hence, $u(r, s)$ is a solution of 
(4.28). Conversely, let $u(r, s)$ be a solution of (4.28). Then

$$
\begin{aligned}
\frac{1}{h}[u(r+h, s)-u(r, s)] & \\
= & \frac{s^{2}}{h} \int_{r}^{r+h} e^{s t}(\tilde{b}(x(t)))^{-1} \int_{t}^{r_{\infty}} e^{-s w} \frac{\tilde{c}(x(w))}{\tilde{b}(x(w))} u(w, s) d w d t \\
= & -\frac{s^{2}}{h} \int_{r}^{r+h} \frac{e^{s t}}{\tilde{b}(x(t))} \cdot\left(\int_{r}^{t} e^{-s w} \frac{\tilde{c}(x(w))}{\tilde{b}(x(w))} u(w, s) d w\right) d t \\
& +\frac{s^{2}}{h} \int_{r}^{r_{\infty}} e^{-s w} \frac{\tilde{c}(x(w))}{\tilde{b}(x(w))} u(w, s) d w \cdot \int_{r}^{r+h} \frac{e^{s t}}{\tilde{b}(x(t))} .
\end{aligned}
$$

As $u(r, s)$ is locally integrable, it is clear that, on letting $h \rightarrow 0$, we obtain

$$
u^{\prime}(r, s)=s^{2} \frac{e^{s r}}{\tilde{b}(x(r))} \int_{r}^{r_{\infty}} e^{-s w} \frac{\tilde{c}(x(w))}{\tilde{b}(x(w))} u(w, s) d w
$$

i.e.,

$$
e^{-s r} \tilde{b}(x(r)) u^{\prime}(r, s)=s^{2} \int_{r}^{r_{\infty}} e^{-s w} \frac{\tilde{c}(x(w))}{\tilde{b}(x(w))} u(w, s) d w .
$$

By a similar argument we also have

$$
\frac{d}{d r}\left(e^{-s r} \tilde{b}(x(r)) \frac{d u}{d r}\right)=-s^{2} e^{-s r} \frac{\tilde{c}(x(r))}{\tilde{b}(x(r))} u(r, s),
$$

and thus $u(r, s)$ is a solution of (4.16). Also, as $u(r, s)$ satisfies (4.28) for all $r$, including $r=0$,

$$
u(r, s)=u(0, s)+s^{2} \int_{0}^{r} \int_{t}^{r_{\infty}} e^{-s(w-t)} \frac{\tilde{c}(x(w))}{\tilde{b}(x(t)) \tilde{b}(x(w))} u(w, s) d w d t .
$$

Hence,

$$
\begin{aligned}
\lim _{r \rightarrow r(\infty)} u(r, s) & =u(0, s)+s^{2} \int_{0}^{r_{\infty}} \int_{t}^{r_{\infty}} e^{-s(w-t)} \frac{\tilde{c}(x(w))}{\tilde{b}(x(t)) \tilde{b}(x(w))} u(w, s) d w d t \\
& =1 .
\end{aligned}
$$

(ii) This follows from the relevant Neumann series expansion in the space of bounded continuous functions on $\left[r_{0}, \infty\right), r_{0}$ suitably large, equipped with the usual supremum norm.

We next consider the behaviour as $n \rightarrow \infty$ of the solutions $\phi_{n}(x, s)$ constructed in §2. Observe that

$$
\begin{gathered}
\left(\beta_{k+1}-\beta_{k}\right) a_{k}=\left(\beta_{k+1}-\beta_{k}\right) \sum_{i=1}^{k}\left(a_{i}-a_{i-1}\right) \\
=\left(\beta_{k+1}-\beta_{k}\right) \sum_{i=1}^{k} \frac{\ln \left(\lambda_{i}\right)}{\alpha_{i}-\beta_{i}} \\
=O(1) \cdot d_{k}\left[\ln \left(\lambda_{k}\right)+\ln \left(\lambda_{k-1}\right) \delta_{k-1} d_{k-1}\right. \\
+\ln \left(\lambda_{k-2}\right)\left(\delta_{k-1} d_{k-1}\right)\left(\delta_{k-2} d_{k-2}\right) \\
\left.+\cdots+\ln \left(\lambda_{1}\right)\left(\delta_{k-1} d_{k-1}\right) \cdots\left(\delta_{1} d_{1}\right)\right] \\
\quad(\text { by }(2.25)-(2.26) \text { and }(2.28)) \\
=O(1) d_{k}\left[\ln \left(\lambda_{k}\right)+o(1)\right], \quad \text { by }(4.1),
\end{gathered}
$$


as $\delta_{r} d_{r} \leqslant \delta^{*}\left|d_{r}\right|$ and $d_{r} \rightarrow 0$ as $r \rightarrow \infty$. Also we have (cf. [4, Lemma 3.5])

LEMMA 4.3. Assume that, for $\sigma>\sigma_{1}, d_{k} \lambda_{k}^{-\sigma} \rightarrow 0$ as $k \rightarrow \infty$ and

$$
\sum_{k=1}^{\infty} \frac{1}{\left(\lambda_{k}^{\sigma}\left|d_{k}^{-1}\right|-1\right)\left(\lambda_{k+1}^{\sigma}\left|d_{k+1}^{-1}\right|-1\right)}<\infty .
$$

Then for each real number $\tilde{\sigma}$ with $\tilde{\sigma}>\sigma_{1}$, one can choose $\tilde{\delta}=\tilde{\delta}(\tilde{\sigma})=\sup \left\{\delta_{r}: r \geqslant 1\right\}$ such that the functions $H_{r}^{(n)}(s)$ and $K_{r}^{(n)}(s)$ defined by (2.64)-(2.65) satisfy $\left|H_{r}^{(n)}(s)\right|$ $\leqslant 2$ and $\left|K_{r}^{(n)}(s)\right| \leqslant 2$ for all $n$ and $r, n \geqslant r+1$, and all complex numbers $s$ with $\operatorname{Re} s \geqslant \tilde{\boldsymbol{\sigma}}$.

Proof. We omit the proof for $H_{r}^{(n)}(s)$ since it is similar to the proof for $K_{r}^{(n)}(s)$ given below. Observe that for $\operatorname{Re}(s) \geqslant \tilde{\sigma}$,

$$
\left|\left(1+\lambda_{r}^{s} / d_{r}\right)^{-1}\right| \leqslant\left(\lambda_{r}^{\tilde{\sigma}}\left|d_{r}^{-1}\right|-1\right)^{-1} .
$$

Consequently, the supremum

$$
C=\sup \left\{\left|\left(1+\lambda_{r}^{s} / d_{r}\right)^{-1}\right|: \operatorname{Re} s \geqslant \tilde{\boldsymbol{\sigma}}, r \geqslant 1\right\}
$$

exists and is finite. Choose $\tilde{\delta}$ so that

$$
\tilde{\delta}\left[C+\sum_{k=1}^{\infty} \frac{1}{\left(\lambda_{k}^{\tilde{\sigma}}\left|d_{k}^{-1}\right|-1\right)\left(\lambda_{k+1}^{\tilde{\sigma}}\left|d_{k+1}^{-1}\right|-1\right)}\right] \leqslant \frac{1}{4} .
$$

Note that

$$
\left|K_{r}^{(r+2)}(s)\right|=\left|1+\frac{\delta_{r+1}}{1+\lambda_{r+1}^{s} d_{r+1}^{-1}}\right| \leqslant 1+\tilde{\delta} C \leqslant 2
$$

by (4.33). Also, assuming $K_{r}^{(m)}(s) \leqslant 2$ for $r+2 \leqslant m \leqslant n-1$, it follows from (2.71) that

$$
\left|K_{r}^{(n)}(s)\right| \leqslant 1+2 \tilde{\delta}\left[C+\sum_{k=1}^{\infty} \frac{1}{\left(\lambda_{k}^{\tilde{\sigma}}\left|d_{k}^{-1}\right|-1\right)\left(\lambda_{k+1}^{\tilde{\sigma}}\left|d_{k+1}^{-1}\right|-1\right)}\right] \leqslant 2
$$

by (4.33) again, Thus, by induction, $\left|K_{r}^{(n)}(s)\right| \leqslant 2$ for all $n$ and $r, n \geqslant r+1$, and all $s, \operatorname{Re} s \geqslant \tilde{\boldsymbol{\sigma}}$.

Now, by (4.30) and (4.1), the series $\Sigma\left(\beta_{k+1}-\beta_{k}\right) a_{k}$ is absolutely convergent. Also, by Lemma 4.3 and (2.76)-(2.77) we can define functions

$$
\begin{aligned}
& \Psi_{r}(s)=\lim _{n \rightarrow \infty} \Psi_{r}^{(n)}(s), \\
& \Phi_{r}(s)=\lim _{n \rightarrow \infty} \Phi_{r}^{(n)}(s)
\end{aligned}
$$

that are analytic in the half-plane $\operatorname{Re} s>\tilde{\sigma}>\sigma_{1}$ provided $\tilde{\delta}=\sup \left\{\delta_{r}: r \geqslant 1\right\}$ satisfies (4.33), which we henceforth assume. Finally, define

$$
\begin{aligned}
U_{r}(s) & =\lim _{n \rightarrow \infty} U_{r}^{(n)}(s) \\
& =E(s) \cdot\left(1+\frac{\left(1+\delta_{r}\right) d_{r+1}}{\lambda_{r+1}^{s}}\right) \cdot\left[\prod_{k=1}^{r+1}\left(1+\frac{d_{k}}{\lambda_{k}^{s}}\right)\right]^{-1},
\end{aligned}
$$




$$
V_{r}(s)=\lim _{n \rightarrow \infty} V_{r}^{(n)}(s)=E(s) \cdot\left[\prod_{k=1}^{r+1}\left(1+\frac{d_{k}}{\lambda_{k}^{s}}\right)\right]^{-1}
$$

On letting $n \rightarrow \infty$ in (2.74)-(2.75), we now have that for fixed $x$ in $\left[a_{r-1}, a_{r}\right]$, $\lim _{n \rightarrow \infty} \phi_{n}(x, s)=\phi(x, s)$, where

(4.38) $\phi(x, s)=A_{r}(s) \exp \left(\alpha_{r} s x\right)+B_{r}(s) \exp \left(\beta_{r} s x\right), \quad x \in\left[a_{r-1}, a_{r}\right]$,

and

$$
\begin{aligned}
& A_{r}(s)=\lambda_{r}^{-s} \exp \left[s \sum_{k=r}^{\infty}\left(\beta_{k+1}-\beta_{k}\right) a_{k}\right] \cdot \frac{w_{r}-w_{r-1}}{w_{\infty}} U_{r}(s) \Psi_{r}(s), \\
& B_{r}(s)=\exp \left[s \sum_{k=r}^{\infty}\left(\beta_{k+1}-\beta_{k}\right) a_{k}\right] \frac{w_{r-1}}{w_{\infty}} V_{r}(s) \Phi_{r}(s) .
\end{aligned}
$$

Let

$$
\psi(x, s)=e^{-\gamma s x} \phi(x, s),
$$

where $\gamma$ is defined in (4.6). Then we have

THEOREM 4.4. (i) The function $\psi(x(r), s)$ is the unique solution of (4.28) for all $s$ in the half-plane $\operatorname{Re} s>\sigma_{0}$.

(ii) If $U_{r}(s)$ and $V_{r}(s)$ are analytic in the half-plane $\operatorname{Re} s>\tilde{\sigma}>\sigma_{1}$ for all $r \geqslant 1$, then, for $\operatorname{Re} s>\tilde{\sigma}>\sigma_{1}$, the function $\psi(x(r), s)$ is a solution of (4.28) if and only if $\psi(x(r), s) \rightarrow 1$ as $r \rightarrow r(\infty)$.

REMARKs. (1) $U_{r}$ and $V_{r}$ are analytic in $\operatorname{Re}(s)>\tilde{\sigma}>\sigma_{1}$ if, for example, $E(s)$ is analytic in $\operatorname{Re}(s)>\sigma_{1}$, and none of the factors $1+d_{n} \lambda_{n}^{-s}$ has a zero in this region, i.e., for all $n \geqslant 1$,

$$
\ln \left|d_{n}\right| / \ln \lambda_{n} \leqslant \sigma_{1} .
$$

(2) Similar results (i.e., Theorems 4.2 and 4.4) apply to the related integral equation

$$
u(r, s)=1+\int_{r}^{r_{\infty}} k_{1}(r, t, s) u(t, s) d t
$$

where

$$
\begin{aligned}
k_{1}(r, t, s) & =-s^{2} e^{-s t} \frac{\tilde{c}(x(t))}{\tilde{b}(x(t))} \int_{r}^{t} \frac{e^{s v}}{\tilde{b}(x(v))} d v, \quad r \leqslant t, \\
& =0, \quad r>t,
\end{aligned}
$$

formed by interchanging the order of integration in (4.28). Here, the analogue of Theorem 4.4(ii) requires a different method of proof (cf. (4.45)).

Proof. (i) By Theorem 4.2 and (4.15) it is enough to show that $\psi(x, s)$ satisfies (4.13) and $\psi(x, s) \rightarrow 1$ as $x \rightarrow \infty$. The proof now follows closely that of [4, Lemmas 4.2 and 4.4].

(ii) Observe that $\sigma_{2}$ (defined in (4.3)) is no larger than $\sigma_{1}$ (defined in (4.31)). As $\psi(x(r), s), \operatorname{Re} s>\sigma_{1}$, is already a solution of (4.16), the result is a consequence of Theorem 4.2(ii). 
As an immediate consequence of Theorem 4.4(i), we have

Corollary 4.5. For any $s, \operatorname{Re} s>\sigma_{0}$, and any constant $C$ the boundary condition $u(r, s) \rightarrow C$ as $r \rightarrow r_{\infty}$ determines a unique solution, $C \psi(x(r), s)$, of the differential equation (4.16).

THEOREM 4.6. Assume (4.41) holds. If there exists a solution $u(r, s)$ of (4.28) analytic for all $s$ in a region $G$ intersecting $B=\left\{s: \operatorname{Re} s>\sigma_{0}\right\}$, and $E(s)$ is analytic in $G$, then $u(r, s)=\psi(x(r), s)$ (and hence $u(r, s) \rightarrow 1$ as $\left.r \rightarrow r_{\infty}\right)$ and $E(s) \neq 0$ for all $s \in G$.

Proof. By Theorem 4.4(i), $u(r, s)=\psi(x(r), s)$ for $s \in B$. Consequently, by uniqueness of analytic continuation, $u(r, s)=\psi(x(r), s)$ for all $s \in G$. But, by (4.36)-(4.37),

$$
\psi(x(r), s)=E(s) \chi(x(r), s),
$$

where $\chi(x(r), s)$ is analytic in a half-plane $\operatorname{Re} s>\tilde{\sigma}>\sigma_{1}$ containing $G$. If $E(s)=0$ for some $s \in G$, then $u(\cdot, s)$ is the zero function, which cannot be a solution of (4.28). Consequently, $E(s) \neq 0$ for $s \in G$.

By way of example, if we consider the case $d_{n}=p_{n}^{-\theta}, \theta>1$ fixed, $\lambda_{n}=p_{n}$ (i.e., (1.4)), then $E(s)=\zeta(s+\theta)(\zeta(2 s+2 \theta))^{-1}$, and $\sigma_{0}+\theta=1, \sigma_{1}+\theta=1 / 2, \sigma_{2}+\theta$ $=0$, while $\delta_{r}>0$ for all $r$. Then Theorem 4.6 is the main theorem in [4]. Observe that, from Theorem 4.4, for $\operatorname{Re}(s+\theta)>1$ we have $\psi(x, s) \rightarrow 1$ as $x \rightarrow \infty$. Consequently, we have the identity

$$
1=\psi(0, s ; \boldsymbol{\delta})+\sum_{n=1}^{\infty}\left\{\psi\left(a_{n}, s ; \boldsymbol{\delta}\right)-\psi\left(a_{n-1}, s ; \boldsymbol{\delta}\right)\right\}
$$

i.e.,

$$
\begin{aligned}
\frac{\zeta(2 s+2 \theta)}{\zeta(s+\theta)}= & \tilde{A}_{1}^{(\infty)}(0, s ; \boldsymbol{\delta}) \\
+ & \tilde{B}_{1}^{(\infty)}(0, s ; \delta) \\
& +\sum_{n=1}^{\infty} \tilde{A}_{n}^{(\infty)}(0, s ; \delta)\left\{e^{\left(\alpha_{n}-\gamma\right) s a_{n}}-e^{\left(\alpha_{n}-\gamma\right) s a_{n-1}}\right\} \\
& \quad+\tilde{B}_{n}^{(\infty)}(0, s ; \delta)\left\{e^{\left(\beta_{n}-\gamma\right) s a_{n}}-e^{\left(\beta_{n}-\gamma\right) s a_{n-1}}\right\}
\end{aligned}
$$

for $\operatorname{Re}(s+\theta)>1$, where $\tilde{A}_{n}^{(\infty)}$ and $\tilde{B}_{n}^{(\infty)}$ are defined in (2.79). A similar formula for $(\zeta(s+\theta))^{-1}$ follows by considering the special case (1.5) mentioned earlier.

Note also that, as the Euler product

$$
E(s)=\prod_{p \text { prime }}\left(1+p^{-s-\theta}\right)
$$

is convergent for $\operatorname{Re}(s+\theta)=1(s+\theta \neq 1)$ by [13, p. 59], it is true that $\psi(x, s) \rightarrow 1$ as $x \rightarrow \infty$ for these values of $s$. Conversely, if one could establish (e.g. by direct asymptotic methods) that (4.13) (or (4.16)) had a solution tending to one as $x \rightarrow \infty$ $\left(r \rightarrow r_{\infty}\right)$ for all $s$ with $\operatorname{Re}(s+\theta)=1(s+\theta \neq 1)$, then Theorems 4.2 and 4.6 show that $E(s) \neq 0$ for these values of $s$. This would provide a differential equation proof of the prime number theorem. 
5. The associated wave equation. We consider here the linear partial differential equation

$$
u_{x x}-\tilde{b}(x) u_{x t}+\tilde{c}(x) u_{t t}=0
$$

for $x, t \geqslant 0$. Observe that for $x$ in $\left[a_{n-1}, a_{n}\right]$,

$$
\tilde{b}^{2}(x)-4 \tilde{c}(x)=\tilde{b}_{n}^{2}\left[1-4 \tilde{c}_{n} / b_{n}^{2}\right]-\tilde{b}_{n}^{2} \text { as } n \rightarrow \infty,
$$

by (4.11)-(4.12). Hence the equation is of hyperbolic type, at least for large $x$. The characteristics through $\left(x_{0}, t_{0}\right)$ are given by

$$
t-t_{0}=\int_{x_{0}}^{x}-\frac{1}{2} \tilde{b}(u)\left\{1+\sqrt{1-4 \tilde{c}(u) \tilde{b}^{-2}(u)}\right\} d u
$$

and

$$
t-t_{0}=\int_{x_{0}}^{x}-\frac{1}{2} \tilde{b}(u)\left\{1-\sqrt{1-4 \tilde{c}(u) \tilde{b}^{-2}(u)}\right\} d u .
$$

It is clear from (4.11)-(4.12) and (4.5) that (eventually) the characteristic (5.2) has negative slope, while from the fact that

$$
1-\sqrt{1-4 \tilde{c} \tilde{b}^{-2}}=\frac{4 \tilde{c}}{\tilde{b}^{2}} \cdot \frac{1}{1+\sqrt{1-4 \tilde{c} \tilde{b}^{-2}}},
$$

it follows that the slope of the characteristic (5.3) depends upon the function $\tilde{c}$; for example, given (2.10), the latter slope is positive if $d_{n}>0$ for all $n$ and negative if $d_{n}<0$ for all $n$. Note also that by (4.11)-(4.12) again, as $n \rightarrow \infty$,

$$
\int_{0}^{a_{n}} \tilde{b}(u) d u=\sum_{r=1}^{n}\left(a_{r}-a_{r-1}\right) \tilde{b}_{r} \sim \ln \left(\lambda_{1} \lambda_{1} \cdots \lambda_{n}\right)
$$

and

$$
\begin{aligned}
\int_{0}^{a_{n}} \frac{\tilde{c}(u)}{\tilde{b}(u)} d u & =\sum_{r=1}^{n}\left(a_{r}-a_{r-1}\right) \cdot \frac{\tilde{c}_{r}}{\tilde{b}_{r}} \\
& =O(1) \cdot \sum_{r=1}^{n} \ln \left(\lambda_{r}\right) \cdot \frac{\beta_{r}-\gamma}{\alpha_{r}-\beta_{r}} \\
& =O(1) \cdot \sum_{r=1}^{n} \ln \left(\lambda_{r}\right) \cdot d_{r}=O(1)
\end{aligned}
$$

by (4.1). Thus, along the characteristics (5.2) if we assume (4.18) it follows from (5.4) that as $x \rightarrow \infty, t \rightarrow-\infty$, while by (5.5) the characteristics (5.3) have the property that as $x \rightarrow \infty, t$ tends to the finite limit

$$
t_{\infty}=t_{0}-\int_{x_{0}}^{\infty} \frac{2 \tilde{c}}{\tilde{b}} \cdot \frac{1}{1+\sqrt{1-4 \tilde{c} \tilde{b}^{-2}}} .
$$

Observe also that if $d_{n}<0$ for all $n$, the characteristic through $\left(0, t_{0}^{*}\right)$, where

$$
2 t_{0}^{*}=\int_{0}^{\infty}\left(\tilde{b}-\sqrt{\tilde{b}^{2}-4 \tilde{c}}\right),
$$

is asymptotic to the $x$-axis as $x \rightarrow \infty$. 
Consider now the change of variable

$$
\xi=\frac{1}{2} \int_{0}^{x} \tilde{b}(u) d u+t
$$

and set $w(x, \xi)=u(x, t)$, where $u$ is any solution of (5.1). Then

$$
w_{\xi \xi}=\frac{4}{\tilde{b}^{2}(x)-4 \tilde{c}(x)} w_{x x}-\frac{2 \tilde{b}^{\prime}(x)}{\tilde{b}^{2}(x)-4 \tilde{c}(x)} w_{\xi} .
$$

Here, as $\tilde{b}$ is a step function, $\tilde{b}^{\prime}$ is an appropriate sum of delta functions. Notice that as $a_{n} \sim K \cdot \ln \left(\lambda_{n}\right) \cdot\left(d_{1} d_{2} \cdots d_{n-1}\right)^{-1}$, as $n \rightarrow \infty$ one can see from (5.4) that in the special cases (1.4) and (1.5) we have (roughly) $\int_{0}^{x} \tilde{b} \approx \theta^{-1} \ln x$, or $\tilde{b}(x) \approx(\theta x)^{-1}$. Consequently, for large $x$,

$$
\frac{4}{\tilde{b}^{2}(x)-4 \tilde{c}(x)}=4 \tilde{b}^{-2}(x)\left(1-\frac{4 \tilde{c}(x)}{\tilde{b}^{2}(x)}\right)^{-1} \approx 4 \theta^{2} x^{2}
$$

Thus, for these cases (5.1) bears some resemblance to the automorphic wave equation (see e.g. [5, (1.8)])

$$
u_{t t}=y^{2}\left(u_{x x}+u_{y y}\right)+\frac{1}{4} u \text {. }
$$

Although the precise connection between (5.1) and the analytic function $E(s)$ is not clear as yet, the following possible application indicates that this connection could be quite useful. The application involves a theorem which is a special case of a result of Müntz [7] and Szász [12] (cf. also [9, 11]). Consider the function sequence $\left\{e^{-\mu_{n} t}\right\}_{n \geqslant 1}$ in $L^{2}(0, \infty)$, where the numbers $\mu_{n}$ are real and positive. This sequence is said to be closed if

$$
\int_{0}^{\infty} f(t) e^{-\mu_{n} t} d t=0, \quad n \geqslant 1,
$$

implies $f$ is zero almost everywhere and complete if any $f$ in $L^{2}(0, \infty)$ can be approximated, in norm, by polynomials

$$
P_{n}(t)=\sum_{k=1}^{n} a_{k} e^{-\mu_{k} t}
$$

It is known (see e.g. [9, p. 26]) that $\left\{e^{-\mu_{n} t}\right\}$ is closed if and only if it is complete. In addition, we have

THEOREM 5.1 (MƯNTZ - SZÁSZ). The sequence $\left\{e^{-\mu_{n} t}\right\}$ is closed if and only if

$$
\sum_{n=1}^{\infty} \frac{1}{\mu_{n}}=\infty
$$

Thus, in particular, if $\sum \mu_{n}^{-1}<\infty$ then $\left\{e^{-\mu_{n} t}\right\}$ is not closed. To prove the latter statement one needs to show that if $\sum \mu_{n}^{-1}<\infty$, then there is a nontrivial function $f(t)$ in $L^{2}(0, \infty)$ whose Laplace transform $F(s)$ vanishes at the prescribed set of points $\left\{\mu_{n}\right\}$. One might attempt to construct such an $f$ as follows. Set

$$
d_{n}=-\mu_{n}^{-1}, \quad \delta_{n}=-\delta<0, \quad \lambda_{n}=\mu_{n}^{1 / \mu_{n}} .
$$


In this case the wave equation (5.1) (with the sign of the middle term reversed) corresponds to the Euler product

$$
E(s)=\prod_{n=1}^{\infty}\left(1-\frac{1}{\mu_{n} \mu_{n}^{-s / \mu_{n}}}\right),
$$

and both sets of characteristic lines (5.2)-(5.3) have positive slope and finite limit values for $t$ as $x$ approaches infinity along the characteristic. Consider the boundary value problem defined by (5.1) and the boundary conditions

$$
\begin{gathered}
u(x, 0)=u_{t}(x, 0)=0, \\
u(0, t)=f(t) .
\end{gathered}
$$

The Laplace transform $U(x, s)$ of the solution $u(x, t)$ of $(5.1),(5.15)-(5.16)$ satisfies (4.9). If one can determine $f$ so that $U(x, s)$ is bounded as $x$ tends to infinity (e.g. by making use of the geometry of the hyperbolic equation), then, by Corollary 4.5 and (4.36)-(4.40), $u(0, t)=f(t)$ transforms to a constant multiple of $E(s)$ which vanishes when $s=\mu_{n}, n \geqslant 1$, as required.

\section{REFERENCES}

1. R. Courant and D. Hilbert, Methods of mathematical physics, Vol. II, Interscience, New York, 1962.

2. M. S. P. Eastham, Theory of ordinary differential equations, Van Nostrand Reinhold, London, 1970.

3. M. Faierman and I. Knowles, On a mixed problem for a hyperbolic equation with a discontinuity in the principal coefficients, Proc. London Math. Soc. (3) 46 (1983), 137-166.

4. I. Knowles, Eigenvalue problems and the Riemann zeta function. II, Proc. Sympos. Differential Equations (Univ. of Dundee), Lecture Notes in Math., vol. 1032, Springer, Berlin, 1983, pp. 267-297.

5. P. Lax and R. Phillips, Scattering theory for automorphic functions, Princeton Univ. Press, Princeton, N. J., 1976.

6. J. Lehner, Lectures on modular forms, Nat. Bur. Standards Appl. Math. Ser. 61, U.S. Government Printing Office, Washington, D.C. 1969.

7. C. H. Müntz, Über den Approximationssatz von Weierstrass, Schwarz's Festschrift, Berlin, 1914, pp. 303-312.

8. A. Ogg, Modular form and Dirichlet series, Benjamin, New York, 1969.

9. R. Paley and N. Weiner, Fourier transforms in the complex domain, Amer. Math. Soc. Colloq. Publ., vol. 19, Amer. Math. Soc., Providence, R. I., 1934.

10. C. Ryavec, The analytic continuation of Euler products with applications to asymptotic formulae, Illinois J. Math. 17 (1973), 608-618.

11. L. Schwartz, Etude des sommes d'exponentielles, Hermann, Paris, 1949.

12. O. Szász, Über die Approximation stetiger Funktionen durch lineare Aggregate von Potenzen, Math. Ann. 77 (1916), 482-496.

13. E. C. Titchmarsh, Theory of the Riemann zeta function, Oxford Univ. Press, Oxford, 1951.

14. The theory of functions, 2nd ed., Oxford Univ. Press, London, 1939.

Department of Mathematics, University of Alabama in Birmingham, Birmingham, Alabama 35294 